\title{
Determinantes y evolución de la motivación de los trabajadores en un contexto de crisis económica. El caso de España
}

\author{
Gabriel Pruneda
}

Universidad de Oviedo. Departamento de Sociología

gcpruneda@gmail.com

Recibido: 12-06-2012

Aceptado: 18-01-2013

\section{Resumen}

La motivación de los trabajadores es un factor de gran relevancia, tanto en su vertiente personal como en la empresarial. El objetivo principal de este estudio es analizar los determinantes de la motivación de los trabajadores en los últimos años, en el contexto de crisis en España. Este trabajo contribuye, además, a dar a conocer el nivel de motivación de la población ocupada. Partiendo de los datos de las oleadas de 2008 a 2010 de la Encuesta de Calidad de Vida en el Trabajo ${ }^{1}$, se emplean modelos logit ordenados para estimar los efectos marginales sobre los niveles de motivación de la población ocupada acerca de diversos factores sociodemográficos y laborales.

Los resultados obtenidos indican que el nivel global medio de motivación no ha experimentado cambios en los últimos años, al contrario de lo que se observa para grupos de trabajadores específicos, como es el caso de quienes tienen una jornada semanal inferior a veinte horas o los extranjeros. Respecto a los determinantes de la motivación, no solo existen diferencias temporales, sino que, además, se observan cambios en función del nivel de estudios, de la categoría profesional o entre asalariados y autónomos, aunque es el salario el que arroja los efectos más significativos. Como conclusiones, se resalta el valor que este trabajo tiene por ser el primero de estas características realizado para España, además de aportar evidencia sobre la motivación en un contexto de decrecimiento económico y de apoyar la tesis que defiende el papel clave del salario como elemento motivador.

Palabras clave: motivación en el trabajo; crisis económica; encuesta de calidad de vida en el trabajo; trabajadores autónomos; responsabilidad; nivel educativo; jornada laboral; salarios.

1. El grado de exactitud o fiabilidad de la información cuantitativa y cualitativa, derivada de la elaboración propia por parte del autor, es de la exclusiva responsabilidad de éste. 
Abstract. Determinants and evolution of worker motivation in a context of economic crisis: The case of Spain

Job motivation is a key factor from both a personal and an organizational perspective. The main objective of this study is to analyze the determinants of workers' motivation in recent years in Spain, and hence in a context of crisis. This work also contributes to the knowledge of the motivational situation of the Spanish workforce. Based on data drawn from the 2008-2010 waves of the Quality of Working Life Survey, ordered logit models are used to estimate the marginal effects of sociodemographic, occupational and organizational variables. The results show that the overall level of motivation has not changed in recent years, contrary to what is found for specific groups of workers, such as those who have a working week of less than twenty hours or foreigners. Regarding the determinants of motivation, not only are there differences along time, but changes can also be observed in terms of education, job category or between waged workers and self-employed individuals. Nevertheless, monthly net income yields the most significant effect. In conclusion, the value of this research is threefold: it is the first of its kind to be conducted for Spain, it provides new evidence on motivation in a context of economic crisis, and its results support the thesis that economic incentives are one of the most powerful motivators.

Keywords: work motivation; economic crisis; quality of working life survey; self-employed workers; responsibility; educational level; working day; income.

\author{
Sumario \\ 1. Introducción y objetivos \\ 2. Antecedentes e hipótesis \\ 3. Datos y fuentes utilizados, \\ metodología y análisis descriptivo \\ 4. Factores explicativos y análisis \\ de resultados \\ 5. Conclusiones \\ Referencias bibliográficas \\ Anexo
}

\title{
1. Introducción y objetivos
}

La motivación se puede definir como el conjunto de procesos psicológicos que provocan el interés y la persistente repetición en el tiempo de acciones voluntarias encaminadas a lograr un objetivo concreto (Mitchell, 1982). A la hora de estudiar la motivación dentro de un contexto laboral, es decir, la motivación de los trabajadores, ésta se puede expresar como el deseo de llevar a cabo altos niveles de esfuerzo para conseguir objetivos organizacionales y lograr, al mismo tiempo, que dicho esfuerzo satisfaga ciertas necesidades individuales (Robbins, 1993). En consecuencia, podremos indicar que la gestión de la motivación implica dirigir los intereses de los trabajadores en la misma dirección que las necesidades de la organización y, por tanto, es esencial distinguir los elementos y los procesos que provocan, dirigen y mantienen la conducta de las personas en el trabajo: qué motiva a los trabajadores y cómo motivarlos.

Tanto las características del empleo como las del individuo son factores que afectan a la motivación. El primer grupo consiste en factores externos al individuo, tales como los contenidos del trabajo, las tareas realizadas, la 
remuneración, la autonomía de que se goza, el uso de habilidades, el ambiente laboral, las condiciones de trabajo, los incentivos o las relaciones interpersonales. En cuanto a las características del individuo, nos encontramos con aspectos básicos, afectivos y cognitivos, que están bajo el control de cada persona, quien busca satisfacer las necesidades de sentirse competente. Entre estas características, tendríamos las necesidades, los objetivos o las expectativas, la autodeterminación, el grado de participación en la toma de decisiones que tiene cada individuo y la actitud de éste hacia el trabajo, es decir, el significado que el trabajo tiene para cada persona en particular. Se trata del papel del empleo como vehículo que posibilita la integración y la participación en la sociedad, lo cual proporciona una identidad personal y social a los individuos (Agulló Tomás, 1998).

Debido a que la actual crisis económica está teniendo un impacto indiscutible en el mercado de trabajo y que, en consecuencia, los factores que se acaban de mencionar experimentan cambios que afectan, directa e indirectamente, a las personas, el objetivo principal de este trabajo es analizar el nivel de motivación de la fuerza laboral en España desde 2008 hasta 2010 y explicar sus determinantes, utilizando para ello como herramienta la Encuesta de Calidad de Vida en el Trabajo (ECVT).

Para ello, en primer lugar, se examinarán algunas de las teorías más consolidadas sobre motivación y se expondrán algunas de las investigaciones más relevantes sobre la motivación de los trabajadores, así como un conjunto de estudios que tienen en cuenta la coyuntura de crisis económica a la hora de analizar la motivación laboral. A partir de esta revisión de la literatura, se establecerán las hipótesis a contrastar. Seguidamente, en la sección de metodología, se revisarán las características y el grado de adecuación de la base de datos utilizada para contrastar las hipótesis y se detallarán las pruebas estadísticas realizadas para estudiar empíricamente los efectos de las variables independientes sobre la dependiente. Se incluirá, asimismo, en esta sección, un análisis descriptivo del nivel de motivación de los trabajadores en España. Por último, se ofrecerá una discusión de los resultados obtenidos a partir de los modelos estimados, tanto de cara a determinar qué variables influyen en la motivación, como la evolución en el tiempo de esta influencia, para, finalmente, exponer unas breves conclusiones.

\section{Antecedentes e hipótesis}

Existen diversas teorías sobre la motivación. Dos de las más conocidas son las formuladas por Maslow y Herzberg ${ }^{2}$. La teoría de la jerarquía de necesidades de Maslow (1943), que, a día de hoy, sigue constituyendo una interpretación clásica del comportamiento, establece que existen cinco grupos básicos

2. Entre otras, sirva mencionar la teoría de la agencia, la teoría de las expectativas, la teoría de la equidad, la teoría de gestión por objetivos o la teoría de las necesidades. Para una síntesis sobre teorías de motivación laboral, véase, por ejemplo, Ramlall (2004). 
de necesidades que las personas buscan satisfacer, por este orden: necesidades fisiológicas (alimento, sueño, sexo), necesidades de seguridad (física, psíquica y económica), necesidades sociales (recibir y proporcionar afecto), necesidades de estima (autoconfianza y autovaloración) y necesidades de autorrealización (crecimiento personal). Según esta teoría, lo que motiva a las personas es el deseo de conseguir o mantener las distintas condiciones que suponen la satisfacción de las necesidades básicas, así como otros deseos más intelectuales. Las implicaciones de esta teoría proporcionaron útiles revelaciones para los mandos de las organizaciones, tales como la conveniencia de encontrar diferentes vías para motivar a los empleados mediante el desarrollo de programas o prácticas dirigidas a satisfacer necesidades emergentes o no satisfechas. Es decir, cuando el concepto de la jerarquía de necesidades se aplica a las organizaciones de trabajo, la implicación obvia para la dirección es que tiene la responsabilidad de crear un clima apropiado en el que los empleados puedan desarrollar su potencial al máximo. De esta manera, una necesidad insatisfecha es un motivador potencial de comportamiento, mientras que una necesidad satisfecha deja de serlo.

Por su parte, la teoría de los dos factores (Herzberg, 1968) sostiene que los factores que conducen a la satisfacción laboral (factores motivacionales, intrínsecos al contenido del trabajo) son distintos de aquéllos que conducen a la insatisfacción (factores higiénicos, extrínsecos al trabajo en sí mismo). Entre los primeros, nos encontramos con el reconocimiento laboral, el logro de objetivos, el trabajo en sí mismo, la responsabilidad o el crecimiento; mientras que, entre los segundos, tendríamos las políticas de empresa, el salario, las relaciones con los compañeros y los estilos directivos. La ausencia de factores motivacionales no produciría insatisfacción, del mismo modo que la existencia de los higiénicos no implicaría satisfacción.

También existen numerosas investigaciones que se han ocupado de estudiar la motivación desde un punto de vista empírico, de esta forma se han complementado las teorías, al aportar evidencias sobre los factores más relevantes a la hora de determinar la motivación de los trabajadores.

Entre ellas, nos encontramos con el artículo de Jurgensen (1978), que concluye que los factores considerados más importantes por los trabajadores de una empresa estadounidense a la hora de clasificar un empleo como bueno son: el tipo de trabajo en sí, la seguridad que ofrece o las posibilidades de promoción. Además, determina que los hombres asignan al salario el quinto puesto, mientras que las mujeres le atribuyen el séptimo. Algunos de estos determinantes se repiten en los resultados obtenidos para EE. UU. por Wiley (1997), al estudiar la importancia de diez factores sobre la motivación para realizar mejor el trabajo, ya que los cinco primeros fueron, en este orden: un buen salario, el reconocimiento de las labores realizadas, la seguridad del empleo, la promoción y el crecimiento dentro de la organización y una ocupación interesante.

Si bien los dos estudios anteriores se basan en autovaloraciones de los empleados sobre los factores que les motivan, Kovach (2001) parte de la base de que quienes toman las decisiones relativas a la gestión de personal pueden tener percepciones distintas. Con el objetivo de aportar evidencia en este senti- 
do, analiza el orden que tanto los unos como los otros dan a diez factores motivacionales tales como un buen salario, seguridad en el empleo, posibilidades de promoción, tareas interesantes o buenas condiciones en el trabajo. Basándose en tres encuestas realizadas en EE. UU. que abarcaban un periodo de 40 años, encuentra diferencias significativas entre las percepciones de los mandos y lo que realmente valoraban los empleados. La tercera de las encuestas, realizada en 1986 y en la que participaron 1.000 empleados y 100 mandos directivos, diferenciaba, además, a los trabajadores en función del sexo, la edad, el nivel salarial y el tipo de trabajo, y mostraba que no solo se aprecian diferencias a lo largo del tiempo, sino también entre distintas categorías laborales. Las conclusiones a las que se llega consisten en que los mandos creen que el dinero es el mayor motivador para los trabajadores, mientras que solo tres de los subgrupos de empleados opinan lo mismo: los menores de 30 años, quienes tienen los salarios más bajos y quienes ocupan los puestos menos cualificados. Además, con la excepción de los dos primeros subgrupos citados, el resto situaba las tareas interesantes en una de las tres posiciones superiores de la clasificación, lo que, según Kovach (2001), implica que la extendida opinión de que el dinero es el principal motivador no es más que un mito.

Se constata, así, que existe una corriente de pensamiento que defiende la idea de que los ingresos no son un factor de motivación de los considerados más relevantes, corriente amparada por una gran variedad de artículos en revistas especializadas y por autovaloraciones de los propios empleados. Sirvan como ilustración, en este sentido, además del texto de Kovach (2001), los ya citados de Herzberg (1968) y Jurgensen (1978). Además, existe una conexión con las postulaciones de Maslow (1943), pues de ellas se desprende que los ingresos solamente están de forma explícita detrás de la satisfacción de los dos primeros grupos de necesidades que establece.

Por su parte, y con el objetivo de aportar evidencias en sentido contrario, Rynes et al. (2004) llevaron a cabo un metaanálisis para demostrar que, generalmente, las encuestas sobre la importancia de diversos factores en la motivación de los empleados arrojan resultados inconsistentes con los estudios del comportamiento real de los mismos. Este estudio concluye que el salario no es ni el único ni el principal factor motivacional para todos. De hecho, apuntan que muchos de los otros factores mencionados por investigadores como Maslow y Herzberg (como pueden ser el trabajo interesante y la participación en la toma de decisiones) son también considerados importantes motivadores por muchos estudiosos del tema. Sin embargo, y en línea con las conclusiones de la mayoría de estudios mencionados, aseguran que el salario tiene un fuerte potencial motivador para la mayoría de trabajadores, y su efectividad real depende de gran variedad de factores individuales y de situación, incluida la forma en que se articula en la práctica.

Como complemento a los trabajos que giran en torno a la valoración independiente de diversos factores motivacionales, otros estudios tratan de identificar el grado de efectividad de diversos tipos de motivación. Tal es el caso de Gagné et al. (2010), quienes probaron en trabajadores canadienses la estructura 
de una escala de motivación laboral desarrollada por ellos mismos, basándose en el marco de la teoría de la autodeterminación. Esta teoría proporciona una conceptualización multidimensional de la motivación, lo cual permite realizar su evaluación, tanto del nivel como del tipo. De esta manera, propone dos clases generales de motivación: intrínseca, consistente en el deseo de hacer algo por el mero hecho de ser interesante y agradable, y extrínseca, la que lleva a hacer algo como instrumento para conseguir un objetivo. Estas razones instrumentales difieren en función del grado de internalización ${ }^{3}$ de la motivación y, en líneas generales, lo que esta investigación muestra es que la motivación autónoma proporciona resultados mucho mejores que la motivación controlada, la cual puede estar asociada a comportamientos no deseados por la organización y no tener un efecto significativo en los resultados buscados por ésta.

Finalmente, ha de añadirse que la literatura existente sobre motivación referida al ámbito español es muy escasa y, además, no es habitual que se ocupe de los determinantes que influyen en la motivación en el trabajo. Una excepción a esto la podemos encontrar en la investigación llevada a cabo por García Sedeño et al. (2003), quienes, a partir de una muestra de 500 participantes en programas de formación ocupacional, observaron que determinados tipos de expectativas, tales como la aceptación y la integración social, el aumento de la competencia profesional o la seguridad afectan positivamente a la motivación laboral ${ }^{4}$.

Una vez abordado el tema de la motivación de los trabajadores en general, nos centraremos en las particularidades durante periodos de crisis económica a través de algunos ejemplos de la literatura más reciente.

\subsection{Motivación laboral en un contexto de crisis económica}

Durante los últimos cuatro años, se ha producido un cambio brusco de ciclo económico, que, en el caso español, afecta sobre todo a jóvenes y a trabajadores temporales. Tal y como apunta Vaquero (2011), las condiciones laborales de los más jóvenes han sufrido un fuerte empeoramiento, puesto que la tasa de paro juvenil ha pasado del $21 \%$ en 2008 al $41 \%$ en 2010 . Durante ese mismo año, la tasa de temporalidad de este grupo se acerca al $60 \%$ y los contratos a tiempo parcial suponen uno de cada tres, con lo cual queda patente la precariedad de este colectivo. Por sectores económicos, se observa principalmente una destrucción de empleo inicial en la construcción y en la industria, mientras que, en las primeras fases, la crisis no incide con la misma intensidad en el sector servicios. Adicionalmente, sabemos que el empleo que se destruye en primer lugar como consecuencia de una recesión económica corresponde a

3. La aceptación de una norma que inicialmente estaba regulada por factores externos, de manera que pasa a regularse de forma interna.

4. Nótese que esta investigación no estudia la motivación de los trabajadores propiamente dicha, sino la que hay detrás de la búsqueda de empleo de los participantes en actividades de formación ocupacional. 
puestos de trabajo que requieren un bajo nivel de cualificación, a los que están asociados niveles de motivación más bajos (como veremos más adelante). En consecuencia, sobreviven en una mayor proporción los empleos cualificados, caracterizados por cuestiones tales como niveles de autonomía superiores o un mayor uso de las habilidades personales, y que podemos entender asociados a niveles de motivación más elevados si los ponemos en relación con los hallazgos de Jurgensen (1978), Wiley (1997), Kovach (2001), Rynes et al. (2004) e incluso Maslow (1943) y Herzberg (1968). Como consecuencia de estos cambios en la composición de la fuerza de trabajo española, podemos encontrar variaciones en el nivel de motivación global, en el que, además, también podría estar influyendo el cambio de ciclo per se.

En este sentido, y al contrario de lo que observamos respecto a la literatura sobre motivación en el empleo en términos generales, las investigaciones existentes que se enmarcan en situaciones de crisis son muy escasas. Esto es así especialmente en el ámbito español, pero también en el europeo e incluso en el de los países pertenecientes a la OCDE ${ }^{5}$. Se han consultado diversos informes que han sido elaborados a partir de encuestas tales como la Encuesta Europea de Calidad de Vida y la Encuesta Europea sobre Condiciones de Trabajo, ambas elaboradas por Eurofound, y la Wage Dynamics Network Survey, elaborada por el Banco Central Europeo. En algunos de ellos se encuentran afirmaciones que relacionan ingresos y motivación, en el sentido de que tanto empleados como empleadores temen el impacto negativo de las restricciones salariales en la motivación de los trabajadores (Eurofound, 2012; Lünnemann y Mathä, 2011). No obstante, las referencias a la motivación laboral son residuales y las mencionadas encuestas no incluyen una pregunta sobre el nivel de motivación en el trabajo.

Entre los escasos estudios existentes, cabría citar, por ejemplo, el de Iqbal y Mehri (2011), quienes estudiaron las repercusiones de la crisis, tanto en mandos intermedios como en empleados de categorías más bajas del sector bancario de Islamabad, concluyendo que la crisis mundial reduce la seguridad del empleo, lo que, a su vez, disminuye la lealtad de los empleados y su motivación.

Por su parte, Tomuletiu et al. (2011) parten del propósito de evaluar el impacto de la crisis mundial en el sistema motivacional del profesorado preuniversitario de Rumanía, así como de identificar los factores subyacentes como elementos fundamentales de la mejora del rendimiento. Basándose en los resultados obtenidos, concluyen que los profesores saben que la responsabilidad o las competencias que tienen representan una fuente de satisfacción

5. En este sentido, cabe mencionar que nos encontramos con una situación muy parecida a la que veremos más adelante a la hora de justificar el motivo que imposibilita la ampliación de la dimensión temporal del presente estudio a los años anteriores a 2008, para, así, poder comparar la situación de la motivación laboral en España con anterioridad a la actual crisis y durante la misma. Así, si, en las oleadas anteriores a 2008 de la ECVT, se incluye el enunciado «Existencia de ambiente de trabajo que motiva a mejorar laboralmente», en la última oleada de la Encuesta Europea sobre Condiciones de Trabajo el enunciado que aparece se puede traducir como: «La organización para la que trabajo me motiva a dar el mejor rendimiento». 
profesional. Asimismo, son conscientes de la importancia de los incentivos económicos, a los que anteponen la seguridad en el empleo. También en un contexto rumano, Casuneanu (2011) analizó la información obtenida en 2009 de altos directivos y mandos intermedios. Entre otros resultados, obtiene que las primas en función de la productividad laboral constituyen el mayor incentivo a la hora de motivar a los empleados, seguidas por las primas no ligadas a la productividad y los cursos de formación de corta duración. Sin embargo, los incentivos económicos no son el motivador más efectivo a largo plazo, pues, pasado un tiempo, los empleados se acostumbran al nuevo nivel de ingresos y los incentivos dejan de llevar asociado el deseo de mejora del rendimiento. Los factores motivacionales más relevantes son la estabilidad del empleo, un salario potencial apropiado, posibilidades de ascenso, un paquete de beneficios estimulante, primas, un puesto atractivo, autoridad, responsabilidad y autonomía y desarrollo profesional. Finalmente, Casuneanu (2011) considera que la fuerza de trabajo en Rumanía comprende la importancia de la estabilidad laboral en el contexto económico actual, ya que las preferencias de un gran número de individuos en busca de empleo son: seguridad, el tipo de trabajo y la oferta salarial. Estos resultados apoyan, por tanto, la corriente de opinión consistente en que el dinero no lo es todo en términos de motivación laboral (Jurgensen, 1978; Kovach, 2001; Rynes et al., 2004; Gagné et al., 2010) y el autor sugiere que los directivos deben centrarse más en incentivos no económicos para mejorar la motivación de los trabajadores.

Con la finalidad de estudiar, dentro de la actual coyuntura económica, diversos factores representativos de las necesidades de los trabajadores en las que se centra la empresa, de las razones para trabajar, de factores motivacionales y de, siguiendo la terminología de Herzberg (1968), factores higiénicos, Kropivšek et al. (2011) realizaron una encuesta a directivos de Eslovenia y Croacia. Sus resultados muestran que se presta más atención a la necesidad de seguridad de los empleados, muy posiblemente como consecuencia del alto nivel de inseguridad empresarial en época de recesión. En cuanto a los factores higiénicos, el salario y las bonificaciones son los más relevantes, seguidos por la seguridad del empleo. Respecto a los factores desmotivadores, los más importantes en el caso esloveno son las reducciones de salario y de jornada, mientras que en el croata lo son los despidos. La opinión predominante en cuanto a las razones para trabajar en tiempos de recesión económica es que no se trabaja para «ser alguien», sino para tener algo, resultado totalmente coherente con el obtenido al evaluar la importancia de la satisfacción de las necesidades, donde se estableció la importancia de la necesidad de seguridad en el empleo, cuya base es «tener algo».

Hitka y Vacek (2010) estudiaron la importancia que trabajadores de varias empresas eslovacas conceden a diversos factores motivacionales relacionados con el puesto de trabajo, tales como la retribución, la duración de la jornada o la autonomía en el empleo. Comparando los resultados obtenidos con anterioridad a la crisis con los obtenidos a finales de 2009, hallaron disminuciones significativas a lo largo del tiempo, con lo cual consideraron la influencia de 
la crisis económica global como la principal razón. De esta manera, concluyen que una consecuencia de los crecientes niveles de desempleo es que las personas valoran más sus ocupaciones y están dispuestas a trabajar a cambio de salarios inferiores y prestaciones menores ${ }^{6}$.

Basándose en datos obtenidos durante los años 2007 a 2009, Hitka y Sirotiakovà (2011) analizaron la motivación de los trabajadores de una empresa eslovaca antes del impacto de la crisis económica en el país y al inicio de la misma. En la misma línea de lo visto en Hitka y Vacek (2010), concluyen que los empleados están dispuestos a trabajar aún ante un empeoramiento de las condiciones laborales, debido a la voluntad de mantener sus puestos de trabajo. Además, llegan a afirmar que la crisis económica mundial fue la razón del aumento en la motivación de los trabajadores, aunque se ha de señalar que esta relación causal no queda suficientemente clara. Adicionalmente, muestran cómo la importancia que los empleados dan a los diversos factores motivacionales estudiados disminuye significativamente bajo los efectos económicos y sociales de la crisis mundial. Del mismo modo, establecen que dichos factores habían variado y que su orden se había visto afectado ${ }^{7}$.

Por otro lado, de cara a estudiar la diferencia entre la motivación de trabajadores de organismos públicos y de empresas privadas, y con la finalidad adicional de contribuir a compensar el hecho de que la mayoría de estudios sobre motivación laboral estaban centrados en épocas de prosperidad económica, Duplessis (1988) realizó una investigación en una provincia argentina durante un marco de crisis. Sin pretender hacer una comparación con los datos referidos a momentos de estabilidad económica, y utilizando como instrumento un cuestionario confeccionado al efecto con el que se medían diversos factores relacionados con el empleo, concluyó que, en recesión, los niveles motivacionales son inferiores en los trabajadores del sector privado que en los del público.

\subsection{Hipótesis}

Con base en esta revisión de la literatura, se establece la relevancia de estudiar las características de la motivación de los trabajadores en España y se sientan los fundamentos para plantear las cuatro hipótesis siguientes.

De acuerdo con los resultados derivados de las investigaciones de Hitka y Vacek (2010) y de Hitka y Sirotiakovà (2011), consistentes en que los trabajadores están interesados en mantener el empleo a pesar del empeoramiento de las condiciones laborales, se establece la siguiente hipótesis general:

Hipótesis I: el nivel de motivación de los trabajadores en España aumenta en tiempos de crisis.

6. Los autores de esta investigación no cuantifican el nivel de motivación, sino que estudian la importancia que los trabajadores conceden a varios factores motivacionales.

7. Ver nota 6 . 
A partir de las conclusiones a las que llegan Herzberg (1968), Wiley (1997) y Casuneanu (2011), respecto a que los puestos que conllevan responsabilidad, autoridad, autonomía y tareas más complejas e interesantes tienen asociados niveles de motivación superiores, se formula la siguiente hipótesis:

Hipótesis II: el grado de responsabilidad y autonomía afecta positivamente al nivel de motivación laboral durante periodos de crisis.

Considerando que la estabilidad en el empleo proporciona niveles motivacionales más elevados, tal y como sostienen Jurgensen (1978), Duplessis (1988), Wiley (1997), García Sedeño et al. (2003), Iqbal y Mehri (2011), Tomuletiu et al. (2011), Kropivšek et al. (2011) y Casuneanu (2011), se propone la siguiente hipótesis:

Hipótesis III: la estabilidad laboral en tiempos de crisis tiene un efecto positivo en la motivación de los trabajadores.

Desde el punto de vista de que los ingresos son un importante elemento motivacional, tal y como apuntan las conclusiones de Wiley (1997), Rynes et al. (2004), Kropivšek et al. (2011) y Casuneanu (2011), aunque no necesariamente sean el principal motivador, en línea con las aportaciones de Gagné et al. (2010) y Tomuletiu et al. (2011), se plantea la siguiente hipótesis:

Hipótesis IV: en un contexto de crisis económica, el nivel de ingresos influye positivamente en la motivación de los trabajadores.

\section{Datos y fuentes utilizados, metodología y análisis descriptivo}

En este apartado, estableceremos, en primer lugar, la adecuación de la base de datos utilizada, para, a continuación, exponer brevemente la elección del modelo de estimación en que se basa la determinación de los factores que influyen en la motivación y, finalmente, efectuar una primera aproximación al nivel de motivación de los trabajadores en España.

Respecto a la muestra utilizada para realizar este trabajo, nos servimos de los datos secundarios procedentes de las oleadas de 2008, 2009 y 2010 de la ECVT del Ministerio de Empleo y Seguridad Social de España ${ }^{8}$. La ECVT se considera la herramienta apropiada para el estudio de la motivación laboral por varias razones. En primer lugar, ha de entenderse como una muestra representativa de la población ocupada española de los citados años, pues su ámbito

8. A pesar de que los primeros efectos de la crisis se pueden observar ya a finales de 2007, el presente análisis no incluye ni esta edición de la ECVT ni las anteriores, debido a que la pregunta sobre el nivel de motivación en el trabajo aparece por primera vez en 2008. Por lo tanto, ésta es la razón por la que no se puede llevar a cabo una comparación entre los niveles de motivación anteriores a la crisis y los existentes durante la misma, lo que sin duda ayudaría a la hora de contextualizar e interpretar los resultados. 
geográfico abarca la totalidad del territorio nacional, con la excepción de las ciudades autónomas de Ceuta y Melilla, mientras que el poblacional lo constituyen los ocupados de 16 años en adelante que residen en viviendas familiares. En segundo lugar, aunque no permite realizar una investigación de carácter longitudinal, debido a que no se sigue a los mismos individuos y, por tanto, no se trata de datos de panel, su condición anual facilita la comparación entre años distintos. Pero, principalmente, la ECVT nos aporta el marco adecuado para desarrollar este estudio, debido a que incluye una pregunta sobre el nivel de motivación en el trabajo, que es nuestra variable dependiente, al mismo tiempo que abarca multitud de aspectos referidos a las condiciones laborales de la población ocupada española, como, por ejemplo: la información sobre el puesto de trabajo, la satisfacción respecto a diversas cuestiones referidas al mismo, el ambiente que se respira en la empresa, las relaciones laborales, la educación y la formación o las actitudes y opiniones.

En lo que se refiere al estudio de la dependencia de la motivación de los trabajadores del resto de variables, se utilizarán modelos logit ordenados, encuadrables dentro del análisis multivariante. Estos modelos se consideran los más adecuados para tener en cuenta las características de nuestra variable dependiente, que es categórica, pero cuyos valores constituyen una escala ordenada. Y precisamente éste es nuestro caso, pues la ECVT mide el nivel de motivación usando para ello una escala de Likert de 11 puntos (0: motivación nula; 10: motivación muy alta). Los resultados se mostrarán en la forma de efectos marginales de cada variable sobre la motivación, explicando en qué medida cada variable contribuye a alcanzar un nivel de motivación determinado en los trabajadores. Así, además de mostrar el efecto positivo o negativo de cada variable sobre la motivación, se cuantificará la magnitud de dicho efecto.

Con el fin de hacer más sencilla la interpretación de resultados, y también como consecuencia de la escasa variabilidad de algunas de las categorías originales de la escala, se ha decidido recodificar la variable dependiente en cuatro tramos, agrupando los niveles de motivación 0-4 en el primero (motivación muy baja), el 5 y el 6 en el segundo (motivación baja), el 7 y el 8 en el tercero (motivación alta) y, por último, el 9 y el 10 en el cuarto (motivación muy alta). Adicionalmente, y de cara al análisis de los efectos marginales, se establece un umbral mínimo de referencia para diferenciar entre los resultados que consideremos más relevantes. En concreto, de cara a esta investigación, entenderemos que los efectos que alcancen el 5\% son lo suficientemente relevantes como para detenernos a analizarlos en profundidad.

\subsection{Análisis descriptivo}

Como primera aproximación a las diferencias y a los cambios en la motivación de los trabajadores en España en 2008-2010, se calcula la media para cada categoría de las variables estudiadas. Como punto de referencia, se ha de señalar que el nivel de motivación para el conjunto de los tres años es de 7,20 
en una escala del 0 (nulo) al 10 (muy alto). Los valores alcanzados son de 7,21 en 2008, 7,17 en 2009 y 7,21 en 2010.

A continuación, se hará mención a los resultados más relevantes, asociados, en primer lugar, a variables que recogen características personales, en segundo lugar, a variables representativas de características del puesto de trabajo y, por último, a variables de características de la empresa.

\subsubsection{Caracteristicas personales}

Las medias de los niveles de motivación correspondientes a variables de índole personal se incluyen en el cuadro 1, donde se puede ver que, por edad, quie-

Cuadro 1. Nivel medio de motivación según características personales. $\mathrm{N}=57.459 .300$ (datos elevados)

\begin{tabular}{|c|c|c|c|c|}
\hline & & 2008 & 2009 & 2010 \\
\hline \multirow[t]{2}{*}{ Sexo } & Mujer & 7,11 & 7,18 & 7,12 \\
\hline & Hombre & 7,28 & 7,17 & 7,28 \\
\hline \multirow[t]{5}{*}{ Edad (años) } & $<25$ & 7,38 & 6,92 & 7,25 \\
\hline & $25-29$ & 7,03 & 7,27 & 7,34 \\
\hline & $30-44$ & 7,19 & 7,19 & 7,13 \\
\hline & $45-55$ & 7,24 & 7,19 & 7,19 \\
\hline & $>=56$ & 7,31 & 7,14 & 7,42 \\
\hline \multirow[t]{2}{*}{ Nacionalidad } & Extranjeros & 6,62 & 7,14 & 7,14 \\
\hline & Españoles & 7,27 & 7,18 & 7,22 \\
\hline \multirow[t]{4}{*}{ Estudios } & $<=$ Primaria & 7,13 & 7,16 & 7,12 \\
\hline & FP & 7,24 & 7,07 & 7,38 \\
\hline & Bachiller & 7,24 & 6,96 & 6,93 \\
\hline & Universitarios & 7,30 & 7,38 & 7,31 \\
\hline \multirow[t]{6}{*}{ Modalidad del hogar } & Unipersonal & 6,91 & 7,01 & 7,18 \\
\hline & Familia monoparental con hijos & 6,85 & 7,02 & 6,79 \\
\hline & Con pareja con hijos & 7,31 & 7,23 & 7,22 \\
\hline & Con pareja sin hijos & 7,16 & 7,16 & 7,22 \\
\hline & Con padres $\mathrm{u}$ otros familiares & 7,20 & 7,13 & 7,25 \\
\hline & Otros & 7,08 & 7,18 & 7,25 \\
\hline \multirow[t]{2}{*}{ Situación laboral de la pareja* } & No ocupada & 7,28 & 7,30 & 7,28 \\
\hline & Ocupada & 7,26 & 7,18 & 7,19 \\
\hline \multirow{6}{*}{$\begin{array}{l}\text { Ingresos mensuales netos } \\
\text { en el hogar }(€)\end{array}$} & $<=1.000$ & 6,92 & 6,84 & 6,87 \\
\hline & $>1.000-1.200$ & 7,13 & 7,17 & 7,19 \\
\hline & $>1.200-1.600$ & 7,25 & 7,07 & 7,23 \\
\hline & $>1.600-2.100$ & 7,10 & 7,16 & 7,17 \\
\hline & $>2.100-3.000$ & 7,24 & 7,21 & 7,18 \\
\hline & $>3.000$ & 7,51 & 7,42 & 7,55 \\
\hline \multirow{2}{*}{$\begin{array}{l}\text { Cambio de residencia } \\
\text { por motivos laborales }\end{array}$} & No & 7,28 & 7,18 & 7,24 \\
\hline & Sí & 6,94 & 7,16 & 7,10 \\
\hline
\end{tabular}

$N^{*}=20.651 .608 \quad N^{* *}=39.540 .341$

Fuente: ECVT 2008-2010. Elaboración propia. 
nes mayor variabilidad presentan son los menores de 25 años. Esto puede ser debido a la incertidumbre laboral que los más jóvenes soportan a causa de la crisis, puesto que es uno de los colectivos más afectados por la misma, como queda de manifiesto con su elevada tasa de paro y temporalidad.

En cuanto a la nacionalidad, observamos que la motivación de los españoles es superior a la media global los tres años y no experimenta grandes cambios, mientras que, aunque la de los extranjeros no llega a alcanzar dicha media, aumenta de forma considerable en 2009 hasta casi lograrlo y se mantiene estable en 2010. Esto podría deberse a que los puestos de trabajo que más se resienten como consecuencia de la crisis son, en su mayoría, los no cualificados, precisamente el tipo de ocupación que desarrolla un gran número de extranjeros. De esta manera, el peso que tienen los trabajadores extranjeros con empleos cualificados (más ricos en funciones por definición y, por tanto, más susceptibles de conllevar un nivel de motivación más elevado), dentro de este colectivo, aumenta a partir de 2009. Dicho de otra manera, la expulsión de ocupados precarios como consecuencia de la crisis afecta en mayor medida a la población extranjera, lo cual hace que el nivel de motivación medio de este colectivo mejore.

Por estudios, los universitarios tienen una motivación superior en todo momento a la media global, lo que puede deberse a que son precisamente quienes más fácilmente acceden a puestos de trabajo cualificados y menos sufren el desempleo, además de tratarse de estudios finalistas con un marcado componente vocacional. Lo contrario sucede para el grupo de trabajadores que tiene estudios primarios o inferiores.

Por modalidad del hogar, son los trabajadores que viven con hijos pero sin pareja quienes presentan niveles de motivación marcadamente inferiores a los del resto. Respecto a los ingresos mensuales netos del hogar, como cabría esperar, los niveles de motivación más bajos corresponden al tramo inferior de ingresos (hasta $1.000 €$ mensuales) y los más elevados, al superior (por encima de $3.000 €$ ). Por último, la diferencia sustancial que se observa en 2008 entre quienes han cambiado de residencia por motivos laborales (por debajo de la media global los tres años) y quienes no lo han hecho (por encima) se reduce notablemente en 2009 y 2010.

\subsubsection{Características del puesto de trabajo}

En el cuadro 2, se incluyen las medias para las variables relativas al puesto de trabajo. Por situación profesional, vemos que son los asalariados temporales del sector privado quienes tienen los niveles de motivación más bajos, inferiores a la media global los tres años. Una de las razones puede ser debida a que son este tipo de puestos los que padecen la crisis con mayor intensidad, como se ha mencionado previamente. En el extremo opuesto, nos encontramos con el colectivo de los empresarios o profesionales, especialmente aquéllos que tienen empleados. La razón, en este caso, podría ser la responsabilidad asociada a haber puesto en marcha un proyecto del que dependen puestos de trabajo. Por tipo de puesto de trabajo, son los empleados sin subordinados los menos 
motivados ${ }^{9}$, con niveles inferiores a la media global los tres años, mientras que los directores, con niveles superiores, son los más motivados, y los encargados y jefes de taller u oficina quienes sufren un descenso motivacional más acusado.

De la CNO-94, se obtiene que los trabajadores no cualificados son los menos motivados, quizás ello está relacionado con que la mayoría de puestos que ocupan habitualmente no se caracterizan por la riqueza de funciones y no requieren una cualificación especial. Dicho caso se sitúa por debajo de la media global los tres años. Los más motivados son los directores, seguidos de técnicos y profesionales científicos e intelectuales, todos ellos puestos cualificados y ricos en funciones.

Respecto a los ingresos mensuales netos, el corte entre quienes tienen niveles de motivación superiores e inferiores a la media global se corresponde con

Cuadro 2. Nivel medio de motivación según características del puesto de trabajo. $\mathrm{N}=57.459 .300$ (datos elevados)

\begin{tabular}{|c|c|c|c|c|}
\hline & & 2008 & 2009 & 2010 \\
\hline \multirow[t]{7}{*}{ Situación profesional } & Asalariado temporal del sector público & 7,37 & 7,34 & 7,38 \\
\hline & Asalariado indefinido del sector público & 7,11 & 7,13 & 7,27 \\
\hline & Asalariado temporal del sector privado & 6,82 & 6,85 & 6,76 \\
\hline & Asalariado indefinido del sector privado & 7,09 & 6,98 & 7,08 \\
\hline & Empresario o profesional con empleados & 8,48 & 8,44 & 8,33 \\
\hline & Empresario o profesional sin empleados & 7,89 & 7,58 & 7,64 \\
\hline & Otros & 7,50 & 7,62 & 7,64 \\
\hline \multirow{5}{*}{$\begin{array}{l}\text { Tipo de puesto } \\
\text { de trabajo* }\end{array}$} & Empleado sin subordinados & 6,95 & 6,94 & 6,99 \\
\hline & Encargado, jefe de taller u oficina o similar & 8,02 & 7,57 & 7,39 \\
\hline & Mando intermedio & 7,26 & 7,24 & 7,21 \\
\hline & Director de empresa, departamento o sucursal & 8,14 & 8,34 & 8,25 \\
\hline & $\begin{array}{l}\text { Ocupado independiente, sin jefes } \\
\text { ni subordinados }\end{array}$ & 7,89 & 7,52 & 7,86 \\
\hline \multirow[t]{8}{*}{ Ocupación (CNO-94) } & Fuerzas armadas & 6,72 & 6,71 & 7,57 \\
\hline & $\begin{array}{l}\text { Directores de las empresas y } \\
\text { de las administraciones públicas }\end{array}$ & 7,86 & 8,08 & 7,90 \\
\hline & $\begin{array}{l}\text { Técnicos y profesionales científicos } \\
\text { e intelectuales }\end{array}$ & 7,54 & 7,59 & 7,55 \\
\hline & Técnicos y profesionales de apoyo & 7,24 & 7,12 & 7,16 \\
\hline & Empleados de tipo administrativo & 6,94 & 6,78 & 6,73 \\
\hline & $\begin{array}{l}\text { Trabajadores de los servicios de restauración, } \\
\text { personales, protección y vendedores de los } \\
\text { comercios }\end{array}$ & 7,36 & 7,25 & 7,39 \\
\hline & $\begin{array}{l}\text { Trabajadores cualificados de la agricultura } \\
\text { y la pesca }\end{array}$ & 7,28 & 6,87 & 7,08 \\
\hline & $\begin{array}{l}\text { Artesanos y trabajadores cualificados de } \\
\text { las industrias manufactureras, la construcción } \\
\text { y la minería }\end{array}$ & 7,26 & 7,05 & 7,36 \\
\hline
\end{tabular}

9. Obsérvese que éste es el colectivo mayoritario dentro de la fuerza de trabajo española (ver anexo). 
Cuadro 2. Nivel medio de motivación según características del puesto de trabajo. $\mathrm{N}=57.459 .300$ (datos elevados) (continuación)

\begin{tabular}{|c|c|c|c|c|}
\hline & & 2008 & 2009 & 2010 \\
\hline \multirow[t]{2}{*}{ Ocupación (CNO-94) } & $\begin{array}{l}\text { Operadores de instalaciones y maquinaria } \\
\text { y montadores }\end{array}$ & 6,96 & 6,92 & 6,74 \\
\hline & Trabajadores no cualificados & 6,50 & 6,64 & 6,57 \\
\hline \multirow{12}{*}{$\begin{array}{l}\text { Ingresos mensuales } \\
\text { netos }(€) \text { por tipo } \\
\text { de jornada }\end{array}$} & $<600$ Parcial & 6,85 & 6,65 & 7,15 \\
\hline & Completa & 6,95 & 6,92 & 6,96 \\
\hline & 600-1.000 Parcial & 6,94 & 6,95 & 6,99 \\
\hline & Completa & 6,91 & 6,98 & 6,85 \\
\hline & $>1.000-1.200$ Parcial & 7,10 & 7,21 & 7,21 \\
\hline & Completa & 7,15 & 7,12 & 7,15 \\
\hline & $>1.200-1.600$ Parcial & 7,36 & 7,65 & 7,55 \\
\hline & Completa & 7,34 & 7,20 & 7,24 \\
\hline & $>1.600-2.100$ Parcial & 7,88 & 7,60 & 7,38 \\
\hline & Completa & 7,42 & 7,35 & 7,55 \\
\hline & > 2.100 Parcial & 8,12 & 7,44 & 7,56 \\
\hline & Completa & 7,71 & 7,76 & 7,62 \\
\hline \multirow[t]{2}{*}{ Tipo de contrato** } & Temporal & 6,94 & 6,98 & 6,91 \\
\hline & Indefinido & 7,10 & 7,02 & 7,13 \\
\hline \multirow{12}{*}{$\begin{array}{l}\text { Antigüedad en la } \\
\text { empresa (años) } \\
\text { por tipo de contrato }\end{array}$} & $<1$ Temporal & 6,86 & 6,93 & 6,78 \\
\hline & Indefinido & 7,67 & 7,60 & 7,56 \\
\hline & 1-2 Temporal & 6,91 & 7,18 & 7,13 \\
\hline & Indefinido & 7,31 & 7,02 & 7,30 \\
\hline & 3-5 Temporal & 7,16 & 7,08 & 6,96 \\
\hline & Indefinido & 7,06 & 7,04 & 7,01 \\
\hline & 6-10 Temporal & 6,99 & 6,71 & 6,84 \\
\hline & Indefinido & 7,14 & 7,17 & 7,19 \\
\hline & 11-20 Temporal & 7,50 & 6,23 & 6,84 \\
\hline & Indefinido & 6,89 & 6,91 & 7,03 \\
\hline & $>20$ Temporal & 6,65 & 7,15 & 6,86 \\
\hline & Indefinido & 6,98 & 6,86 & 7,11 \\
\hline \multirow{6}{*}{$\begin{array}{l}\text { Tiempo semanal } \\
\text { de trabajo (horas) }\end{array}$} & $<=20$ & 7,29 & 6,69 & 7,30 \\
\hline & $>20-30$ & 7,22 & 7,11 & 7,14 \\
\hline & $>30-<40$ & 7,06 & 7,08 & 6,94 \\
\hline & 40 & 7,20 & 7,21 & 7,23 \\
\hline & $>40-45$ & 7,31 & 7,24 & 7,27 \\
\hline & $>45$ & 7,29 & 7,37 & 7,37 \\
\hline \multirow[t]{2}{*}{ Duración de la jornada } & Parcial & 7,04 & 6,93 & 7,15 \\
\hline & Completa & 7,24 & 7,22 & 7,22 \\
\hline \multirow{3}{*}{$\begin{array}{l}\text { Convenio o estatuto } \\
\text { específico de } \\
\text { regulación }^{\star \star}\end{array}$} & No & 7,17 & 7,13 & 7,20 \\
\hline & Sí & 6,91 & 6,88 & 6,94 \\
\hline & No sabe & 7,07 & 7,01 & 7,06 \\
\hline \multirow{2}{*}{$\begin{array}{l}\text { Puesto acorde } \\
\text { con la formación }\end{array}$} & No & 6,28 & 6,19 & 6,14 \\
\hline & Sí & 7,48 & 7,45 & 7,49 \\
\hline
\end{tabular}

$\left.N=57.335 .964\left(^{*}\right) \quad N=47.049 .843^{(*}\right) \quad N=56.172 .197\left(^{(\star \star}\right)$

Fuente: ECVT 2008-2010. Elaboración propia. 
la cantidad de $1.200 €$. El grupo con una mayor variabilidad lo constituyen quienes no alcanzan los $600 €$ y tienen jornada parcial, posiblemente debido a la mayor inestabilidad laboral asociada a este tipo de puestos. Además, vemos que tener un contrato indefinido conlleva un nivel de motivación superior al de los empleados temporales. En este sentido, puede apuntarse la mayor seguridad de mantener el puesto de trabajo como una de las razones que podría haber detrás, aunque ambos colectivos están por debajo de la media global ${ }^{10}$.

Según la antigüedad y diferenciando en función de la duración de la relación laboral, el caso más llamativo es el de los indefinidos que llevan en su puesto menos de un año, ya que son la única categoría cuya motivación está por encima de la media los tres años.

Otros resultados destacables son el hecho de que la motivación es estable y superior a la media en el caso de quienes tienen jornada completa, que la ausencia de convenio o estatuto específico de regulación conlleva niveles motivacionales superiores respecto a su existencia ${ }^{11} \mathrm{y}$, finalmente, que la motivación es superior a la media global para puestos acordes con la formación e inferior en caso contrario.

\subsubsection{Características de la empresa}

El cuadro 3 recoge variables relacionadas con las características de la empresa y muestra que la relación es inversa entre el nivel de motivación y el tamaño del centro de trabajo, y es inferior a la media global a partir de las 10 personas. Algo muy similar ocurre con el tamaño de la empresa, a excepción de las dos categorías superiores. Entre las razones, podrían estar la variedad de tareas en el puesto y la cercanía con los dirigentes de la organización.

En resumen, no se observa una variación sustancial del nivel de motivación en términos globales, aunque podemos destacar los siguientes cambios como los más relevantes:

- En 2009, se aprecia una tendencia al descenso del nivel de motivación y sucede lo contrario en 2010. De todos modos, las variaciones conjuntas son muy leves ${ }^{12}$.

- Si comparamos 2008 tanto con 2009 como con 2010, el mayor cambio en el nivel de motivación se da en los extranjeros $(+0,52)$.

- En el periodo 2009-2010, los mayores cambios en el nivel de motivación tienen lugar en los trabajadores con una jornada semanal de 20 horas como máximo $(+0,61)$ y en los temporales con unos ingresos mensuales netos inferiores a $600 €(+0,50)$.

Son, por tanto, las variables duración de la jornada semanal, tipo de contra-

10. La duración del contrato (indefinido o temporal) solo afecta a los trabajadores por cuenta ajena.

11. Únicamente los trabajadores por cuenta ajena responden a esta pregunta.

12. Recordemos que, en una escala del 0 al 10, en 2008, el nivel global de motivación es 7,21; en $2009,7,17$, y en $2010,7,21$. 
Cuadro 3. Nivel medio de motivación según características de la empresa: medias. $\mathrm{N}=57.459 .300$ (datos elevados)

\begin{tabular}{llccc}
\hline & & 2008 & 2009 & $\mathbf{2 0 1 0}$ \\
\hline Tamaño del centro & 1 persona & 7,68 & 7,51 & 7,51 \\
de trabajo & 2-9 personas & 7,42 & 7,33 & 7,37 \\
& 10-49 personas & 7,11 & 7,07 & 7,10 \\
& 50-249 personas & 6,80 & 6,91 & 6,99 \\
& $>=250$ personas & 6,77 & 6,81 & 6,85 \\
\hline Tamaño de la empresa & 1 persona & 7,72 & 7,52 & 7,64 \\
& 2-9 personas & 7,53 & 7,52 & 7,54 \\
& 10-49 personas & 7,15 & 7,08 & 7,01 \\
& 50-249 personas & 6,89 & 6,91 & 6,95 \\
Actividad económica & Agricultura, ganadería y pesca & 6,96 & 6,97 & 7,04 \\
de la empresa (CNAE-09) & Industria & 6,95 & 6,75 & 6,64 \\
& Construcción & 7,00 & 6,91 & 7,06 \\
& Comercio & 7,27 & 7,41 & 7,35 \\
& Transporte & 7,40 & 7,25 & 7,42 \\
& Hostelería & 7,12 & 6,85 & 6,89 \\
& Administración pública, educación y sanidad & 7,38 & 7,11 & 7,34 \\
& Otros servicios & 7,35 & 7,41 \\
& 7,15 & 7,18 & 7,07 \\
\hline
\end{tabular}

Fuente: ECVT 2008-2010. Elaboración propia.

to, nacionalidad e ingresos mensuales netos las que parecen verse más afectadas durante esta crisis en España. Además, se encuentran al alza los cambios en los niveles de motivación asociados a ellas. En este sentido, podemos, asimismo, decir que es muy posible que estos aumentos de motivación estén interrelacionados entre sí, ya que todos ellos apuntan a puestos de trabajo de baja calidad.

\section{Factores explicativos y análisis de resultados}

En este apartado, se muestran los resultados obtenidos a partir de las estimaciones efectuadas para, en primer lugar, determinar si las medias globales del nivel de motivación en España a lo largo de los años estudiados son estadísticamente distintas y, en segundo lugar, analizar cuáles son los factores determinantes del nivel de motivación.

Así, debido a que las medias globales de cada año son muy próximas entre sí (7,21 en 2008, 7,17 en 2009 y 7,21 en 2010), se estima oportuno llevar a cabo un contraste de igualdad de medias para determinar si las diferencias son estadísticamente significativas. La herramienta utilizada con este fin es el test de Wald, cuyos resultados se muestran en el cuadro 4.

Como se puede apreciar, los resultados no nos permiten rechazar la hipótesis nula consistente en que las medias son iguales, por tanto, debemos rechazar a un nivel de significatividad del $1 \%$ la hipótesis I propuesta al final de 
Cuadro 4. Contraste de igualdad de medias y test de Wald $(n=24.393)$

\begin{tabular}{|c|c|c|c|c|}
\hline \multirow{2}{*}{$\frac{\text { Año }}{2008}$} & \multirow{2}{*}{$\begin{array}{l}\text { Media } \\
7,211\end{array}$} & \multirow{2}{*}{$\begin{array}{c}\text { D. t. } \\
0,033\end{array}$} & \multicolumn{2}{|c|}{ [I. C. 95\%] } \\
\hline & & & 7,145 & 7,277 \\
\hline 2009 & 7,173 & 0,035 & 7,105 & 7,241 \\
\hline 2010 & 7,209 & 0,039 & 7,133 & 7,285 \\
\hline \multicolumn{3}{|c|}{ Test de Wald } & \multicolumn{2}{|c|}{ Test de Wald } \\
\hline \multicolumn{3}{|c|}{$[\mathrm{mot}] 2008-[\mathrm{mot}] 2009=0$} & \multicolumn{2}{|c|}{$[\mathrm{mot}] 2009-[\mathrm{mot}] 2010=0$} \\
\hline \multicolumn{3}{|c|}{$F(1,24.392)=0,63$} & \multicolumn{2}{|c|}{$F(1,24.392)=0,49$} \\
\hline \multicolumn{3}{|c|}{ Prob $>F=0,4282$} & \multicolumn{2}{|c|}{ Prob $>F=0,4818$} \\
\hline
\end{tabular}

Fuente: ECVT 2008-2010. Elaboración propia.

la primera sección de este trabajo (el nivel de motivación de los trabajadores aumenta en tiempos de crisis).

Como ya se ha mencionado, los resultados de las estimaciones se presentan a través de los efectos marginales. Éstos informan sobre el aumento o la disminución de la probabilidad de que un determinado grupo de trabajadores se encuentre en uno de los tramos de motivación, siempre con respecto a la probabilidad asociada a la categoría que previamente se ha tomado como referencia y que, por tanto, no se habrá incluido en dicha estimación. A modo de ejemplo, obsérvese que, en 2008, la probabilidad de los trabajadores de nacionalidad distinta a la española de tener un nivel de motivación muy alto disminuye un $6,6 \%$ respecto a la de los españoles ${ }^{13}$ (ver el cuadro 5).

Por último, se estima interesante mencionar que se ha introducido una variable que recoge la tasa de paro anual para cada comunidad autónoma, a modo de control de la coyuntura económica. De la interpretación de los efectos marginales asociados (prácticamente nulos), se desprende que son los factores micro y no los macro los que explican el nivel de motivación en el trabajo, lo que no impide que los segundos ejerzan influencia en los primeros.

Con el objetivo de determinar la evolución temporal de los efectos de las distintas variables en la motivación, se llevan a cabo estimaciones individuales para cada año. Así podremos detectar si, al adentrarnos en una coyuntura económica de crisis, hay variables que comienzan o dejan de producir efectos en la motivación. Por motivos de simplicidad y claridad en la presentación, se incluyen exclusivamente los resultados correspondientes a los tramos de motivación muy baja y muy alta (los extremos), tal y como se observa en el cuadro 5.

Siguiendo el mismo orden del análisis descriptivo, comenzaremos con las variables representativas de aspectos sociodemográficos.

13. Nótese que esta lectura es válida para la mayoría de las variables estudiadas por ser categóricas, pero no para las continuas. 


\subsection{Caracteristicas personales}

A pesar de que el efecto marginal de la edad es de escasa magnitud, indica que la probabilidad de tener un nivel de motivación muy bajo aumenta anualmente un $0,4 \%$. Por tanto, el nivel de motivación será más alto en jóvenes que en personas mayores ${ }^{14}$.

Respecto a la nacionalidad, vemos que, en 2008 , la probabilidad de que los extranjeros estén muy motivados disminuye en un $6,6 \%$ con respecto a la de los españoles, mientras que ni en 2009 ni en 2010 se observan diferencias relevantes. Esto ratifica lo expuesto en la sección de análisis descriptivo, donde se ha señalado que la motivación de los extranjeros aumentó considerablemente en 2009, casi equiparándose a la de los españoles, tanto en este año como en 2010. La explicación la podemos encontrar de nuevo si nos fijamos en la tipología de los puestos de trabajo destruidos, pues esta destrucción ha afectado en mayor proporción a los trabajadores extranjeros y, por tanto, sería de esperar que quienes conservan su empleo estén más motivados. En otras palabras, los efectos de la crisis estarían condicionando estos resultados a través de la expulsión de población ocupada precaria.

Los efectos más significativos corresponden a los estudios. En este sentido, la probabilidad de tener una motivación muy alta es mayor para los individuos que no tienen estudios o los tienen primarios que para los universitarios. Además esa probabilidad aumenta progresivamente $(5,1 \%$ en $2008,8,1 \%$ en 2009 y $8,6 \%$ en 2010).

En vista de estos resultados, y teniendo en cuenta que las medias globales de motivación son mayores para quienes tienen estudios superiores, como se ha señalado en la sección de análisis descriptivo, se puede apuntar como posible explicación el subempleo de algunos universitarios ${ }^{15} \mathrm{y} / \mathrm{o}$ que éstos sean más exigentes a la hora de autodefinirse muy motivados por tener expectativas más elevadas en términos de puesto de trabajo, ingresos, responsabilidad o riqueza de las funciones desempeñadas, entre otros. Así, aunque la información que se desprende a partir del análisis de las medias indica que los universitarios están más motivados, los efectos marginales nos estarían diciendo que no es el hecho de tener una titulación universitaria lo que determina un nivel de motivación superior, sino las mejores condiciones laborales de que este colectivo disfruta en términos generales. En otras palabras, la mayor motivación de los universitarios explicada en la sección anterior vendría determinada por su acceso a puestos de mayor responsabilidad, estabilidad y salario. En la medida en que no tuvieran estas condiciones laborales, su nivel de motivación sería incluso inferior al de las personas con bajos niveles de cualificación, probablemente asociado

14. La edad es una de las dos variables continuas, la otra es la antigüedad, como veremos más adelante.

15. En este sentido, y a partir de la oleada de 2003 de la ECVT, Gobernado Arribas (2007) establece que el 2,1\% de la población ocupada en España eran universitarios en los dos estratos ocupacionales inferiores, quienes, además, ganaban lo mismo, o incluso menos, que el resto de ocupados en estos estratos. 


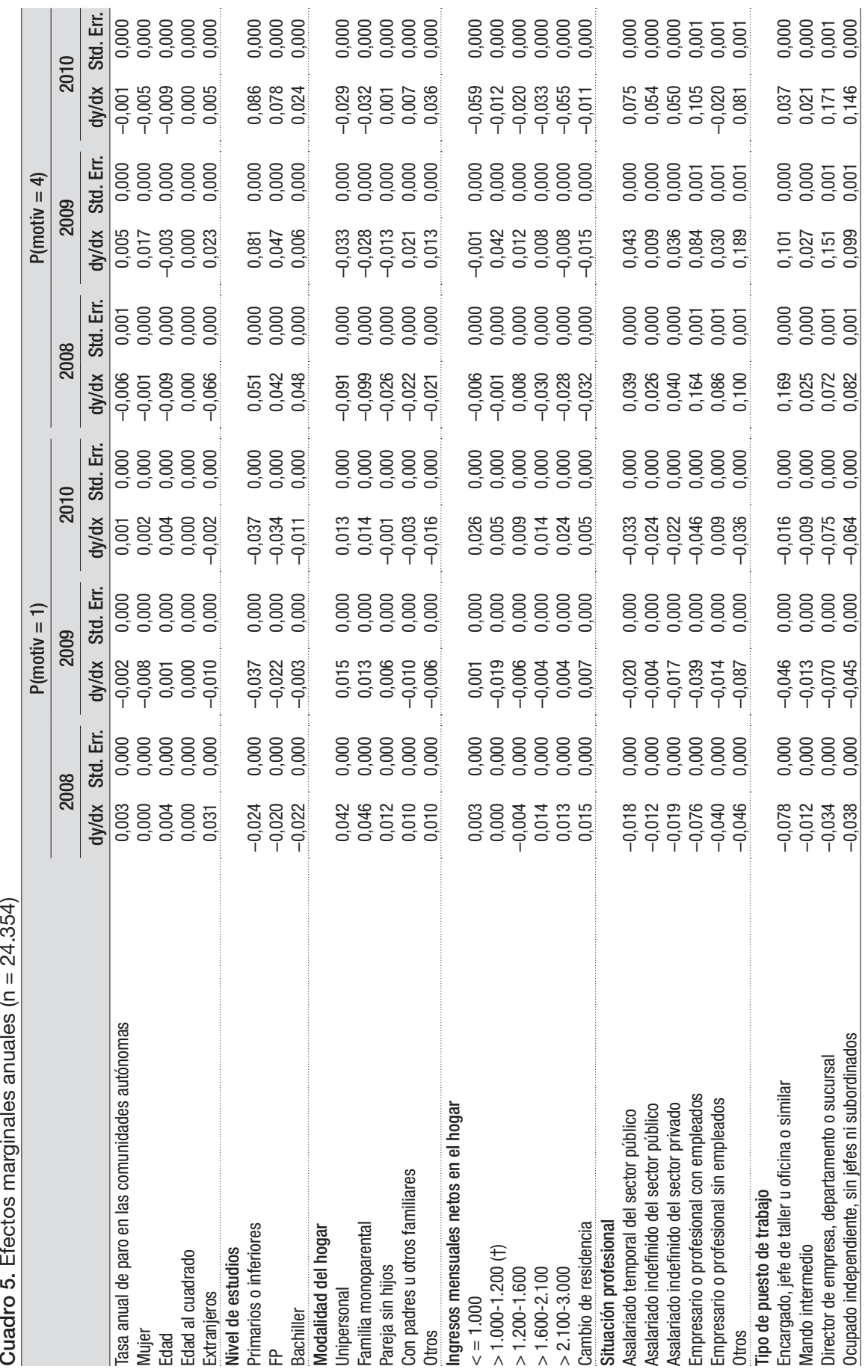


을

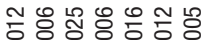
OTOT,

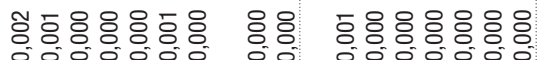

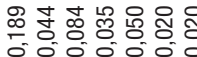
훙ㅎㅇㅇㅎㅎㅎㅇㅇㅇㅇ

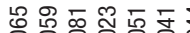
i००000

\section{ㅎㅇㅇㅇㅇㅇㅎㅎㅇ}

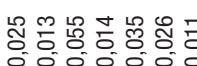

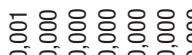

๓ 음 íííi

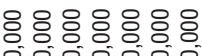
00000

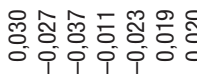
흥ㅇㅇㅇㅇㅇ응 000000
응응

\%ั

0

ஃ 웡 융응 용용 00000

흥ㅇㅇㅇㅇㅇㅇㅇㅇ

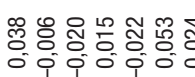

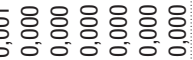

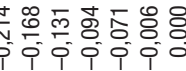

- 8088808

000000

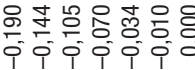

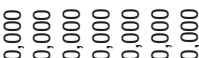

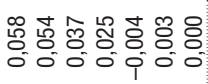

흥ㅇㅇㅇㅇㅇㅇㅡ.

10

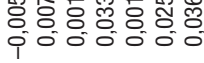

등ㅇㅇㅇㅇㅇㅇㅇㅇ

0.0 00000

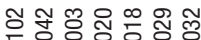

000.00

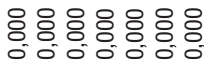

10

승 응용홍음 잉응

i

용ㅇㅇ

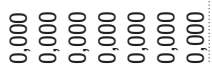

양융융영영

응

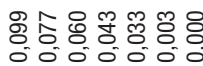

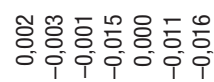

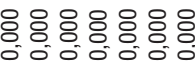

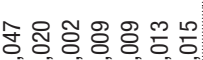

ip i i o

응ㅇㅇㅇㅇㅇㅇ

용 ஜ

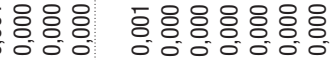

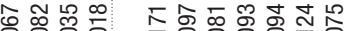

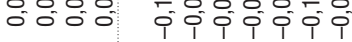

응용흥ㅇㅇㅇㅇㅇㅇㅇㅇㅇㅎํ

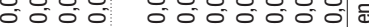

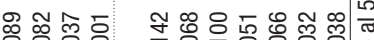
O- 0 - i

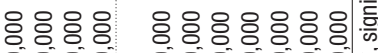
0000000000

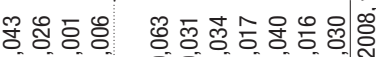
ipió 0000000

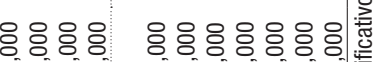
000000000 .0

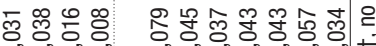

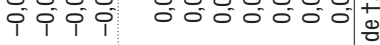

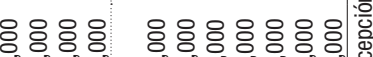
00000000000

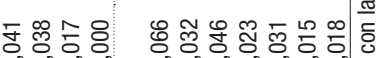
i i

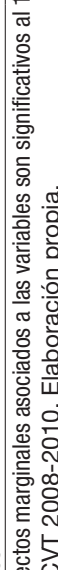


a unas altas expectativas derivadas precisamente de su nivel de estudios. Esta circunstancia estaría, por tanto, poniendo de manifiesto la segmentación del mercado laboral español.

Muy similar es el caso de aquéllos que tienen estudios de formación profesional, entre los cuales aumenta la probabilidad correspondiente hasta alcanzar un $7,8 \%$ en 2010 . Estos hechos pueden atribuirse a que la destrucción de empleo a causa de la crisis ha sido mucho más intensa entre quienes no tienen una titulación universitaria, de manera que quienes conservan su empleo posiblemente experimenten un aumento de su nivel de motivación precisamente como consecuencia de esto. Por otra parte, también puede deberse a que, al desaparecer los empleos más precarios, los efectos marginales asociados a este colectivo sean más elevados, tal y como se ha visto en el caso de los extranjeros, aunque del mismo modo se debería considerar que fueran los más motivados inicialmente.

Respecto a la modalidad del hogar, en 2008, quienes viven solos y quienes viven únicamente con sus hijos tienen una probabilidad de estar muy motivados que disminuye en un $9,1 \%$ y en un $9,9 \%$, respectivamente, en relación con la de quienes viven con su pareja e hijos. Sin embargo, en ninguno de los dos años sucesivos, los efectos marginales son destacables. Así, podemos establecer que, mientras que, al comienzo de la crisis, la modalidad del hogar era relevante a la hora de determinar el nivel de motivación, deja de serlo a partir de 2009.

El caso contrario ocurre con los ingresos mensuales netos del hogar, pues solamente en 2010 observamos efectos marginales relevantes. Concretamente, disminuye un 5,9\% la probabilidad de que las personas con ingresos que no superan los $1.000 €$ tengan una motivación muy alta respecto a los hogares que ingresan más de 3.000. Estos resultados son los que se podrían esperar a priori, pues es habitual que los miembros de una misma familia se apoyen entre sí y, en 2010, la destrucción de empleo debida a la crisis ya había incrementado sustancialmente el número de parados de larga duración. La similitud entre los niveles de motivación de trabajadores pertenecientes a hogares con ingresos bajos (no superiores a $1.000 €$ ) y los que forman parte de hogares con ingresos más elevados (más de $2.100 €$ ) puede ser debida a que los hogares unipersonales tendrán un importante peso entre los primeros.

\subsection{Características del puesto de trabajo}

Tomando como categoría de referencia la de los asalariados temporales del sector privado para el análisis de la situación profesional, se observa que, en 2010, el resto de grupos de trabajadores presenta un nivel de motivación muy superior ${ }^{16}$. Por lo tanto, no se rechaza la hipótesis III, que establece que la estabilidad en el empleo tiene un impacto positivo en el nivel de motivación de los

16. Como excepción a esto, nos encontramos con los autónomos sin empleados, lo que podría deberse a que la salida sustancial de este colectivo del mercado de trabajo produce un efecto negativo sobre los niveles de motivación asociados. 
trabajadores. Por otra parte, tanto en 2008 como en 2009, el único colectivo cuya motivación sobresale de manera considerable es el de los empresarios o profesionales con empleados. Esto podría deberse al hecho de que quienes tienen empleados a su cargo son, en gran medida, los verdaderos emprendedores (a diferencia de una parte importante de autónomos sin empleados, que pueden buscar el autoempleo ante la falta de alternativas en el trabajo asalariado), y constataría lo expuesto en el análisis descriptivo. Así, la situación profesional gana peso en el marco de crisis a la hora de determinar la motivación de los ocupados.

Lo mismo sucede con el tipo de puesto de trabajo. Tomando como referencia en este caso a los empleados sin asalariados, se observa que los efectos en la motivación son crecientes en el tiempo para los trabajadores con cargos directivos y para los ocupados independientes sin jefes ni subordinados. Estos puestos suelen ser más ricos en funciones, conllevar una alta responsabilidad y requerir de la toma de decisiones frecuente, con lo cual es necesaria la implicación de la persona y están asociados a cierto éxito profesional previo que demuestra la capacidad y el interés por desempeñar el trabajo. Por el contrario, los empleados sin subordinados pueden ser, en muchos casos, trabajadores que desarrollan un trabajo concreto, no porque sea la ocupación a la que aspiran, sino por el simple hecho de la necesidad de tener un empleo o porque no tienen la cualificación necesaria ni están preparados para ejercer una mayor responsabilidad. Confirmamos así los incrementos en la motivación a medida que aumenta la responsabilidad asociada al puesto de trabajo y observamos, además, que esto tiene un efecto creciente a lo largo del periodo analizado. Por lo tanto, no se rechaza la hipótesis II, consistente en que el grado de responsabilidad y autonomía en el trabajo tiene un impacto positivo en la motivación laboral.

La repercusión de la ocupación según la CNO-94 en el nivel de motivación es menor en 2008. Tomando a los empleados de tipo administrativo como referencia, los resultados más relevantes corresponden a los técnicos y profesionales científicos e intelectuales, cuya motivación va en aumento. El razonamiento de estas diferencias iría en la misma línea que el que se acaba de señalar para el tipo de puesto de trabajo.

El mayor impacto en la motivación lo tienen los ingresos mensuales netos. Con base en sus efectos marginales, la probabilidad de que la motivación sea muy alta se incrementa a medida que lo hacen los ingresos. Tal y como se puede ver, el efecto aumenta en 2009 para, en 2010, caer incluso por debajo de los niveles de 2008. La razón de este descenso podría estar en que, en 2010, las consecuencias de la crisis son más severas que el año anterior, puesto que el desempleo pasa del 18,01\% al 20,06\% (INE, 2012), y los trabajadores podrían estar valorando más el hecho de tener empleo y atribuir una importancia inferior al nivel de ingresos ${ }^{17}$. Para ilustrar esto, y teniendo en cuenta que la categoría de referencia la constituyen los ingresos superiores a $2.100 €$, obsérvese

17. Como veremos más adelante, los efectos marginales de quienes tienen un contrato indefinido aumentan progresivamente en el periodo 2008-2010. 
la diferencia sustancial existente entre dicha categoría y la que comprende los ingresos más bajos (inferiores a $600 €$ ), para la que la probabilidad de tener una motivación muy alta disminuye un 19,0\% en 2008, un 21,4\% en 2009 y un $13,1 \%$ en 2010 . Se demuestra, así, la relación positiva entre motivación e ingresos, pues, cuanto más elevados son éstos, mayor es la probabilidad de que la motivación sea, a su vez, elevada, lo que implica la especial relevancia de esta variable a la hora de explicar el nivel de motivación de los trabajadores. En consecuencia, nuestros resultados apoyan a los de Wiley (1997) y Rynes et al. (2004), y no se rechaza la hipótesis IV, consistente en que el nivel de ingresos tiene un impacto positivo en la motivación de los trabajadores.

Como se ha mencionado con anterioridad, hemos utilizado una variable continua para medir el efecto de la antigüedad en la empresa. Sus efectos marginales son muy leves e indican que la probabilidad de situarse en el tramo inferior de motivación aumenta un $0,5 \%$ por año. Por tanto, el nivel de motivación será más elevado entre las personas con menor antigüedad.

Por su parte, la duración de la jornada semanal deja de tener una influencia relevante a medida que la coyuntura económica empeora, pues sí influye en 2008, pero no tanto en 2009 ni en 2010. Tomando como referencia a quienes trabajan más de cuarenta horas y un máximo de cuarenta y cinco, vemos que, en 2008, la probabilidad de que quienes tienen una jornada semanal inferior a veinte horas estén muy motivados aumenta un $10,2 \%$, mientras que en ninguno de los años posteriores la diferencia es relevante. Esto puede ser debido a que prima el hecho en sí de tener trabajo sobre la calidad del empleo.

El efecto de la duración de la jornada en la motivación sorprende por su cambio de signo. Si bien en 2008 la probabilidad de tener un nivel de motivación muy elevado aumenta para los trabajadores con jornada completa, en 2009 ocurre lo contrario y en 2010 este efecto es aún mayor, pues la probabilidad de que quienes tienen jornada parcial estén muy motivados pasa de un $2,5 \%$ a un $5,3 \%$. De esta manera, podemos concluir que, durante la crisis, el efecto de las dos modalidades de duración de la jornada sobre la motivación se invierte.

\subsection{Características de la empresa}

En la medida en que el nivel de motivación aumenta cuando disminuye el tamaño del centro de trabajo, los resultados derivados de esta variable ratifican lo expuesto en la sección de aspectos descriptivos. Existe una relación inversa entre el tamaño del centro de trabajo y el nivel de motivación, lo que podríamos achacar al hecho de que cuando, en el día a día, se trabaja con un número reducido de personas, el grado de implicación con los compañeros es superior, la información y el conocimiento que se tienen sobre la situación del negocio también lo son y la variedad de tareas en los puestos de trabajo suele ser superior, lo cual se convierte, generalmente, en una mayor implicación en el trabajo. Sin embargo, al no desprenderse ningún patrón temporal de dicha relación, a la hora de contextualizar la evolución en un marco de crisis, 
la interpretación no es muy clara. Esto puede estar relacionado con que son muchas más las empresas que disminuyen su tamaño que las que lo aumentan, así como con que la motivación de los trabajadores, si se producen nuevas contrataciones, no se ve afectada del mismo modo que si tienen lugar despidos.

Finalmente, analizaremos cómo afecta la actividad de la empresa u organización (según la CNAE-09) a la motivación de los trabajadores en España, tomando como referencia la Administración pública, la sanidad y la educación. Los trabajadores con una mayor probabilidad de estar muy motivados son precisamente los que conforman la categoría de referencia, pues los resultados muestran disminuciones en las distintas probabilidades de que el resto se sitúe en este nivel. Detrás de los niveles de motivación superiores de los trabajadores de la sanidad, así como de los de la educación, puede estar el componente vocacional asociado a estas actividades. También podría deberse a que, en muchos casos, estamos hablando del sector público, donde tanto la seguridad como las condiciones de los empleos son, en términos generales, mejores que las del sector privado ${ }^{18}$. Respecto a la evolución en el tiempo, 2009 es el año en que los efectos de esta variable son más intensos y 2008, en el que son menores. Un resultado interesante nos muestra que, para la construcción, la diferencia disminuye a lo largo de los años, que los trabajadores de la hostelería tienen la mayor variabilidad y las mayores diferencias corresponden a los trabajadores del sector primario, para quienes la probabilidad de estar muy motivados disminuye un $14,2 \%$ en 2008 , un $17,1 \%$ en 2009 y un $14,3 \%$ en 2010 .

\section{Conclusiones}

La motivación de los trabajadores es un factor de gran relevancia, tanto en su vertiente personal como en la empresarial, tal y como ha quedado manifiesto a raíz de la revisión de la literatura existente. Tras este repaso, se planteó como objetivo de este estudio analizar los cambios en la motivación de los trabajadores en España dentro del marco de la actual crisis económica, así como estudiar sus determinantes. Para ello, se propusieron cuatro hipótesis que se contrastaron mediante la estimación de modelos logit ordenados a partir de los microdatos de las oleadas de 2008 a 2010 de la ECVT.

Basándonos en los datos obtenidos, se ha podido determinar que la importancia de aspectos como la nacionalidad, la modalidad del hogar o la duración de la jornada semanal disminuye a medida que la crisis se alarga en el tiempo a la hora de explicar el nivel de motivación de los trabajadores. Por el contrario, aumenta la relevancia de cuestiones como los ingresos netos del hogar, en el sentido de que cuanto más elevados son, mayor es la probabilidad de tener una motivación alta, o la situación profesional.

Entre los resultados más decisivos, se quiere destacar que se ha podido verificar el hecho de que la variable que explica en mayor medida la motivación

18. En relación con la existencia de niveles de seguridad en el empleo, más elevados en el sector público respecto al privado, estos resultados estarían apoyando una vez más la hipótesis III. 
de los trabajadores es la que mide el nivel de ingresos netos, de modo que se observa de manera clara la existencia de una relación positiva entre ambas. Así, se ha comprobado que cuanto mayor es el nivel de ingresos, más elevada es la motivación laboral, lo cual corrobora la hipótesis IV y apoya los resultados obtenidos por Wiley (1997), Rynes et al. (2004), Kropivšek et al. (2011) y Casuneanu (2011).

Otro resultado que llama la atención es el hecho de que la probabilidad de disfrutar de una motivación muy alta es mayor para quienes no tienen estudios, los tienen primarios o de formación profesional, que para los universitarios. Además, las diferencias se acentúan al alargarse la crisis.

En lo que se refiere al cambio en el nivel de motivación global de la fuerza de trabajo española, tras contrastar la igualdad entre las medias globales en 2008 (7,21) y en 2009 (7,17), así como las de 2009 y $2010(7,21)$, los resultados obtenidos indican que las diferencias no son significativas. En consecuencia, se demuestra que el nivel medio de motivación no sufre cambios importantes a lo largo del periodo estudiado y, por tanto, se debe rechazar la hipótesis I, consistente en que la motivación de los trabajadores aumenta en tiempos de crisis. Esto significa que nuestros resultados no apoyan las conclusiones a las que llegaron Hitka y Vacek (2010) y Hitka y Sirotiakovà (2011) para el caso eslovaco, consistentes en que la crisis económica produce aumentos en los niveles de motivación. Asimismo, podemos corroborar lo dicho en cuanto a la falta de claridad en la conexión que hacen entre el aumento de motivación y la disminución observada en la importancia que los trabajadores asignan a los múltiples factores motivacionales. Sin embargo, nuestros resultados sí apoyan lo que estos autores ponían de manifiesto en cuanto a que los factores que influyen en la motivación no son exactamente los mismos al inicio de la crisis que al cabo de cierto tiempo, así como que su importancia relativa experimenta variaciones.

Hemos visto también que el tipo de puesto es otra variable que cobra relevancia cuando la inestabilidad económica se hace más patente, lo que se nota especialmente en el aumento del nivel de motivación de quienes desempeñan puestos directivos y con un alto nivel de autonomía. Y teniendo en cuenta que, en función de la situación profesional, el colectivo que presenta niveles de motivación superiores a lo largo del periodo estudiado es el de los empresarios o profesionales con empleados, corroboramos lo que encontraron Herzberg (1968), Wiley (1997) y Casuneanu (2011). Por tanto, podemos aceptar la hipótesis II, consistente en que los trabajadores con puestos directivos tienen niveles de motivación superiores a los individuos en otro tipo de puestos.

En función de dicha situación profesional, se observa que, en un primer momento de nuestro análisis estadístico (oleada de 2008), las únicas diferencias relevantes respecto a los temporales del sector privado estaban en el colectivo de empresarios y profesionales (que tenían estos niveles motivacionales superiores). Sin embargo, a medida que la recesión económica se va agudizando, también el resto de asalariados presenta una motivación significativamente mayor. De forma añadida, nuestros resultados apoyan las conclusiones de Duplessis 
(1988), al mostrar niveles de motivación superiores en los empleados del sector público que en los del privado, con lo cual estos resultados presentan una segunda lectura asociada al grado de estabilidad del empleo. Complementariamente, se observa que la probabilidad de tener una motivación muy alta aumenta en el caso de los contratos indefinidos del sector privado con respecto a los temporales. Como consecuencia de todo lo anterior, nuestros resultados están en la línea de las conclusiones de Jurgensen (1978), Wiley (1997), Iqbal y Mehri (2011), Tomuletiu et al. (2011), Kropivšek et al. (2011) y Casuneanu (2011). No rechazamos, por tanto, la hipótesis III.

Este estudio general sobre los determinantes del nivel de motivación en España debería considerarse como un punto de partida necesario previo a la profundización en el tema, mediante, por ejemplo, estudios pormenorizados que diferencien entre diversos colectivos, tales como sector público y privado, hombres y mujeres, asalariados y autónomos, o distinguiendo en función del nivel de estudios o del tipo de contrato. Adicionalmente, y con el fin de disponer de una perspectiva más amplia en el tiempo que permita estudiar la evolución de la motivación durante un periodo de crisis más amplio, se añadirán los datos de futuras oleadas de la ECVT. Esto supondrá atajar una de las limitaciones de esta primera aproximación exploratoria, ya que, al no disponer de datos correspondientes a más de tres años, no es posible obtener una trayectoria lo suficientemente precisa de la evolución de la motivación laboral en España.

A modo de conclusión final, se quiere destacar que, mediante el presente artículo, se ha podido demostrar que la motivación de la fuerza de trabajo española experimenta cambios a lo largo de los tres años analizados. Además, se han encontrado resultados interesantes tales como el aumento de la probabilidad de tener una motivación muy alta en el caso de aquellos trabajadores con estudios primarios o de FP con respecto a los universitarios. Asimismo, se quiere hacer especial hincapié en el hecho de que los resultados obtenidos son acordes con la tesis que sostiene que los ingresos son uno de los factores motivacionales más potentes, lo cual cuestiona, para el caso español, la que afirma lo contrario. En este sentido, una vez dibujado el mapa de la motivación de los trabajadores en España, sería necesario llevar a cabo investigaciones adicionales sobre esta cuestión, para estudiar qué otro tipo de factores estarían influyendo en dicha motivación, para, de esta manera, establecer qué políticas pueden llevar a cabo las organizaciones, a fin de aumentar la motivación de su plantilla.

\section{Referencias bibliográficas}

Agulló Tomás, E. (1998). «La centralidad del trabajo en el proceso de construcción de la identidad de los jóvenes: Una aproximación psicosocial». Psicothema, 10 (1), 153-165.

Casuneanu, C. (2011). «The Romanian employee motivation system: An empirical analysis". International Journal of Mathematical models and Methods in Applied Science, 5 (5), 931-938. 
Duplessis, J. E. (1988). «Crisis económica y motivación laboral». En: Psicología, trabajo, organización y nuevas formas de empleo: 1er Congreso Iberoamericano y $3^{\circ}$ Nacional de Psicología del Trabajo y de las Organizaciones. Madrid: Colegio Oficial de Psicólogos de Madrid, 231-243.

EUROFOUND (2012). Wages and working conditions in the crisis. European Foundation for the Improvement of Living and Working Conditions.

Gagné, M.; Forest, J.; Gilbert, M.; Aubé, C.; Morin, E. y Malorni, A. (2010). "The motivation at work scale: Validation evidence in two languages». Educational and Psychological Measurement, 70 (4), 628-646.

<http://dx.doi.org/10.1177/0013164409355698>

García Sedeño, M. A.; Barbero García, M. I.; Ávila Carretero, I. y García TEjerA, M. A. (2003). «La motivación laboral de los jóvenes en su primer empleo». Psicothema, 15 (1), 109-113.

Gobernado Arribas, R. (2007). «La sobreeducación en España: Estudio descriptivo y revisión crítica del concepto». Papers: Revista de Sociología, 86, 11-31.

HerzBerg, F. (1968). «One more time: how do you motivate employees?». Harvard Business Review, 46 (1), 53-62.

HitKa, M. y SirotiakOvÀ, M. (2011). "The impact of economic crisis on the change in motivation of furniture company employees - Case study». Drewno: prace naukowe, doniesienia, komunikaty (Wood: research papers, reports, announcements), 54 (185), 119-126.

HiTKA, M. y VACEK, V. (2010). "Changes in motivation of workers in a production company as a result of the economic crisis». MVK Human Potential Development: Search for Opportunities in the New EU States. Vilna, Lituania: Mykolas Romeris University. PMid:21374924

INE (2012). Encuesta de Población Activa [en línea]. <http://www.ine.es>.

IQBAL, M. J. y MEHRI, M. (2011). «Economic crisis and its impact on job motivation and job security: A case of banking sector». Interdisciplinary Journal of Contemporary Research in Business, 1 (3), 104-114.

JuRGENSEN, C. E. (1978). «Job preferences (What makes a job good or bad?)». Journal of Applied Psychology, 63 (3), 267-276. <http://dx.doi.org/10.1037/0021-9010.63.3.267>

Kovach, K. A. (2001). "What motivates employees?: Workers and supervisors give different answers». Business Horizons, 30 (5), 58-65. <http://dx.doi.org/10.1016/0007-6813(87)90082-6>

KropivŠEK, J.; Jelač́č, D. y GrošELJ, P. (2011). «Motivating employees of Slovenian and Croatian wood-industry companies in times of economic downturn». Drvna Industrija, 62 (2), 97-103. <http://dx.doi.org/10.5552/drind.2011.1040>

LÜNNEMANN P. y MATHÄ, T. Y. (2011). «How do firms adjust in a crisis?: Evidence from a survey among Luxemburg firms». Banque Centrale du Luxembourg. Working paper, 50.

Maslow, A. H. (1943). "A theory of human motivation». Psychological Review, 50, 370-396. Reeditado en 2004 como apéndice de GoBle, F. The Third Force: The Psychology of Abraham Maslow. Chapel Hill, NC: Maurice Bassett Publishing. <http://dx.doi.org/10.1037/h0054346>

Mitchell, T. R. (1982). «Motivation: new direction for theory, research and practice». The Academy of Management Review, 7 (1), 80-88. <http://dx.doi.org/10.5465/AMR.1982.4285467> <http://dx.doi.org/10.2307/257251> 
Ramlall, S. (2004). "A review of employee motivation theories and their implications for employee retention within organizations». Journal of American Academy of Business, 5 (1/2), 52-63.

RobBins, S. P. (1993). Organizational behavior. 6a ed. New Jersey: Prentice Hall.

Rynes, S. L.; Gerhart, B. y MinetTe, K. A. (2004). "The importance of pay in employee motivation: Discrepancies between what people say and what they do". Human Resource Management, 43 (4), 381-394.

Tomuletiu, E.; Pop, A.; David, D.; Solovastru, A. y Buicu, G. (2011). "The impact of the world financial crisis on Romanian educational system: Case study on teacher's professional motivation». Procedia Social and Behavioral Sciences, 15, 1497-1501. <http://dx.doi.org/10.1016/j.sbspro.2011.03.318>

VAquero, A. (2011). «Los jóvenes, el acceso al empleo y la formación». Revista Economistas, 6.

WILEY, C. (1997). "What motivates employees according to over 40 years of motivational surveys». International Journal of Manpower, 18 (3), 263-280. <http://dx.doi.org/10.1108/01437729710169373> 


\section{Anexo}

Estadísticos descriptivos. N (2008-2010) $=57.459 .300$ (datos elevados)

\begin{tabular}{|c|c|c|c|c|c|c|c|c|}
\hline & \multicolumn{2}{|c|}{$2008-2010$} & \multicolumn{2}{|c|}{2010} & \multicolumn{2}{|c|}{2009} & \multicolumn{2}{|c|}{2008} \\
\hline & Frec. & d. t. & Frec. & d. t. & Frec. & d. t. & Frec. & d. t. \\
\hline \multicolumn{9}{|l|}{ Sexo } \\
\hline Mujer & 0,433 & 0,496 & 0,443 & 0,497 & 0,437 & 0,496 & 0,422 & 0,494 \\
\hline Hombre & 0,567 & 0,496 & 0,557 & 0,497 & 0,563 & 0,496 & 0,578 & 0,494 \\
\hline \multicolumn{9}{|l|}{ Edad (años) } \\
\hline Media & & 420,3 & & 420,8 & & 410,8 & & 420,1 \\
\hline$<25$ & 0,076 & 0,265 & 0,065 & 0,246 & 0,073 & 0,260 & 0,090 & 0,286 \\
\hline $25-29$ & 0,124 & 0,330 & 0,116 & 0,320 & 0,123 & 0,329 & 0,133 & 0,339 \\
\hline $30-44$ & 0,447 & 0,497 & 0,452 & 0,498 & 0,448 & 0,497 & 0,442 & 0,497 \\
\hline $45-55$ & 0,247 & 0,431 & 0,260 & 0,439 & 0,249 & 0,432 & 0,234 & 0,424 \\
\hline$>=56$ & 0,105 & 0,306 & 0,107 & 0,309 & 0,106 & 0,308 & 0,102 & 0,302 \\
\hline \multicolumn{9}{|l|}{ Nacionalidad } \\
\hline Extranjeros & 0,102 & 0,303 & 0,107 & 0,309 & 0,104 & 0,305 & 0,097 & 0,296 \\
\hline Españoles & 0,898 & 0,303 & 0,893 & 0,309 & 0,896 & 0,305 & 0,903 & 0,296 \\
\hline \multicolumn{9}{|l|}{ Estudios } \\
\hline$<=$ Primaria & 0,386 & 0,487 & 0,344 & 0,475 & 0,403 & 0,490 & 0,408 & 0,491 \\
\hline $\mathrm{FP}$ & 0,216 & 0,411 & 0,247 & 0,431 & 0,196 & 0,397 & 0,205 & 0,404 \\
\hline Bachiller & 0,136 & 0,343 & 0,138 & 0,344 & 0,137 & 0,344 & 0,133 & 0,339 \\
\hline Universitarios & 0,263 & 0,440 & 0,271 & 0,445 & 0,264 & 0,441 & 0,254 & 0,435 \\
\hline \multicolumn{9}{|l|}{ Modalidad del hogar } \\
\hline Unipersonal & 0,063 & 0,242 & 0,063 & 0,243 & 0,062 & 0,240 & 0,063 & 0,244 \\
\hline Familia monoparental con hijos & 0,039 & 0,194 & 0,038 & 0,192 & 0,045 & 0,208 & 0,035 & 0,183 \\
\hline Con pareja y con hijos & 0,486 & 0,500 & 0,495 & 0,500 & 0,480 & 0,500 & 0,482 & 0,500 \\
\hline Con pareja y sin hijos & 0,155 & 0,362 & 0,161 & 0,368 & 0,155 & 0,362 & 0,148 & 0,355 \\
\hline Con padres u otros familiares & 0,184 & 0,388 & 0,171 & 0,377 & 0,173 & 0,379 & 0,206 & 0,404 \\
\hline Otros & 0,073 & 0,261 & 0,071 & 0,256 & 0,084 & 0,278 & 0,066 & 0,248 \\
\hline \multicolumn{9}{|l|}{ Situación laboral de la pareja* } \\
\hline No ocupada & 0,381 & 0,486 & 0,385 & 0,487 & 0,370 & 0,483 & 0,388 & 0,487 \\
\hline Ocupada & 0,619 & 0,486 & 0,615 & 0,487 & 0,630 & 0,483 & 0,612 & 0,487 \\
\hline \multicolumn{9}{|l|}{ Ingresos mensuales netos del hogar $(€)$} \\
\hline$<=1.000$ & 0,107 & 0,309 & 0,115 & 0,319 & 0,102 & 0,302 & 0,104 & 0,306 \\
\hline$>1.000-1.200$ & 0,118 & 0,322 & 0,116 & 0,321 & 0,109 & 0,312 & 0,126 & 0,332 \\
\hline$>1.200-1.600$ & 0,174 & 0,379 & 0,175 & 0,380 & 0,165 & 0,371 & 0,181 & 0,385 \\
\hline$>1.600-2.100$ & 0,208 & 0,406 & 0,214 & 0,410 & 0,210 & 0,407 & 0,199 & 0,399 \\
\hline$>2.100-3.000$ & 0,229 & 0,420 & 0,231 & 0,421 & 0,235 & 0,424 & 0,223 & 0,416 \\
\hline$>3.000$ & 0,165 & 0,371 & 0,149 & 0,356 & 0,179 & 0,384 & 0,167 & 0,373 \\
\hline \multicolumn{9}{|l|}{ Cambio de residencia por motivos laborales } \\
\hline No & 0,793 & 0,405 & 0,801 & 0,399 & 0,788 & 0,409 & 0,790 & 0,408 \\
\hline Sí & 0,207 & 0,405 & 0,199 & 0,399 & 0,212 & 0,409 & 0,210 & 0,408 \\
\hline \multicolumn{9}{|l|}{ Situación profesional } \\
\hline Asalariado temporal del sector público & 0,047 & 0,211 & 0,048 & 0,214 & 0,050 & 0,219 & 0,042 & 0,202 \\
\hline Asalariado indefinido del sector público & 0,159 & 0,365 & 0,147 & 0,355 & 0,171 & 0,376 & 0,157 & 0,364 \\
\hline Asalariado temporal del sector privado & 0,149 & 0,356 & 0,154 & 0,361 & 0,134 & 0,340 & 0,157 & 0,364 \\
\hline Asalariado indefinido del sector privado & 0,465 & 0,499 & 0,464 & 0,499 & 0,448 & 0,497 & 0,482 & 0,500 \\
\hline Empresario o profesional con empleados & 0,046 & 0,209 & 0,045 & 0,208 & 0,055 & 0,228 & 0,038 & 0,190 \\
\hline Empresario o profesional sin empleados & 0,121 & 0,326 & 0,127 & 0,333 & 0,125 & 0,331 & 0,112 & 0,315 \\
\hline Otros & 0,015 & 0,120 & 0,014 & 0,119 & 0,017 & 0,131 & 0,012 & 0,109 \\
\hline
\end{tabular}


Estadísticos descriptivos. N (2008-2010) = 57.459.300 (datos elevados) (continuación)

\begin{tabular}{|c|c|c|c|c|c|c|c|c|}
\hline & \multicolumn{2}{|c|}{ 2008-2010 } & \multicolumn{2}{|c|}{2010} & \multicolumn{2}{|c|}{2009} & \multicolumn{2}{|c|}{2008} \\
\hline & Frec. & d. t. & Frec. & d.t. & Frec. & d. t. & Frec. & d. t. \\
\hline \multicolumn{9}{|l|}{ Tipo de puesto de trabajo** } \\
\hline Empleado sin subordinados & 0,665 & 0,472 & 0,669 & 0,470 & 0,649 & 0,477 & 0,677 & 0,468 \\
\hline Encargado, jefe de taller u oficina o similar & 0,057 & 0,232 & 0,064 & 0,245 & 0,052 & 0,222 & 0,055 & 0,228 \\
\hline Mando intermedio & 0,103 & 0,304 & 0,101 & 0,301 & 0,109 & 0,312 & 0,100 & 0,301 \\
\hline Director de empresa, departamento o sucursal & 0,068 & 0,252 & 0,065 & 0,247 & 0,076 & 0,265 & 0,064 & 0,244 \\
\hline $\begin{array}{l}\text { Ocupado independiente, sin jefes } \\
\text { ni subordinados }\end{array}$ & 0,106 & 0,308 & 0,100 & 0,300 & 0,114 & 0,318 & 0,104 & 0,305 \\
\hline \multicolumn{9}{|l|}{ Ocupación (CNO-94) } \\
\hline Fuerzas armadas & 0,005 & 0,068 & 0,002 & 0,047 & 0,006 & 0,079 & 0,005 & 0,073 \\
\hline $\begin{array}{l}\text { Dirección de las empresas } \\
\text { y de las administraciones públicas }\end{array}$ & 0,075 & 0,263 & 0,072 & 0,259 & 0,081 & 0,273 & 0,071 & 0,256 \\
\hline $\begin{array}{l}\text { Técnicos y profesionales científicos } \\
\text { e intelectuales }\end{array}$ & 0,149 & 0,356 & 0,154 & 0,361 & 0,150 & 0,357 & 0,144 & 0,351 \\
\hline Técnicos y profesionales de apoyo & 0,146 & 0,353 & 0,150 & 0,357 & 0,154 & 0,361 & 0,134 & 0,341 \\
\hline Empleados de tipo administrativo & 0,080 & 0,272 & 0,071 & 0,258 & 0,067 & 0,249 & 0,102 & 0,302 \\
\hline $\begin{array}{l}\text { Trabaj. serv. restaur., pers., protecc. } \\
\text { y vended. ccios. }\end{array}$ & 0,168 & 0,373 & 0,182 & 0,385 & 0,165 & 0,371 & 0,157 & 0,364 \\
\hline $\begin{array}{l}\text { Trabajadores cualificados } \\
\text { de la agricultura y la pesca }\end{array}$ & 0,037 & 0,189 & 0,040 & 0,195 & 0,037 & 0,190 & 0,034 & 0,182 \\
\hline $\begin{array}{l}\text { Artesanos y trabajadores cualificados } \\
\text { de las industrias manufactureras, } \\
\text { la construcción y la minería }\end{array}$ & 0,142 & 0,349 & 0,136 & 0,343 & 0,136 & 0,342 & 0,154 & 0,361 \\
\hline $\begin{array}{l}\text { Operadores de instalaciones y maquinaria } \\
\text { y montadores }\end{array}$ & 0,085 & 0,278 & 0,082 & 0,274 & 0,081 & 0,273 & 0,091 & 0,287 \\
\hline Trabajadores no cualificados & 0,114 & 0,318 & 0,111 & 0,314 & 0,123 & 0,328 & 0,109 & 0,312 \\
\hline \multicolumn{9}{|l|}{ Ingresos mensuales netos (€) } \\
\hline$<600$ & 0,083 & 0,276 & 0,087 & 0,281 & 0,093 & 0,291 & 0,070 & 0,256 \\
\hline $600-1.000$ & 0,233 & 0,423 & 0,226 & 0,418 & 0,231 & 0,422 & 0,240 & 0,427 \\
\hline$>1.000-1.200$ & 0,218 & 0,413 & 0,210 & 0,407 & 0,216 & 0,412 & 0,227 & 0,419 \\
\hline$>1.200-1.600$ & 0,230 & 0,421 & 0,241 & 0,427 & 0,216 & 0,411 & 0,233 & 0,422 \\
\hline$>1.600-2.100$ & 0,136 & 0,342 & 0,136 & 0,343 & 0,135 & 0,341 & 0,137 & 0,343 \\
\hline$>2.100$ & 0,101 & 0,301 & 0,101 & 0,301 & 0,109 & 0,311 & 0,093 & 0,291 \\
\hline \multicolumn{9}{|l|}{ Tipo de contrato*** } \\
\hline Temporal & 0,239 & 0,426 & 0,249 & 0,432 & 0,229 & 0,420 & 0,238 & 0,426 \\
\hline Indefinido & 0,761 & 0,426 & 0,751 & 0,432 & 0,771 & 0,420 & 0,762 & 0,426 \\
\hline \multicolumn{9}{|l|}{ Antigüedad en la empresa (años) } \\
\hline Media & & 11,0 & & 11,4 & & 10,7 & & 10,9 \\
\hline$<1$ & 0,121 & 0,326 & 0,112 & 0,316 & 0,110 & 0,313 & 0,138 & 0,345 \\
\hline $1-2$ & 0,169 & 0,375 & 0,138 & 0,345 & 0,175 & 0,380 & 0,191 & 0,393 \\
\hline $3-5$ & 0,182 & 0,386 & 0,188 & 0,391 & 0,185 & 0,388 & 0,175 & 0,380 \\
\hline $6-10$ & 0,182 & 0,386 & 0,205 & 0,404 & 0,185 & 0,388 & 0,158 & 0,365 \\
\hline $11-20$ & 0,186 & 0,389 & 0,192 & 0,393 & 0,183 & 0,387 & 0,184 & 0,388 \\
\hline$>20$ & 0,160 & 0,366 & 0,165 & 0,371 & 0,161 & 0,367 & 0,154 & 0,361 \\
\hline \multicolumn{9}{|l|}{ Tiempo semanal de trabajo (horas) } \\
\hline$<=20$ & 0,066 & 0,249 & 0,071 & 0,256 & 0,076 & 0,266 & 0,053 & 0,223 \\
\hline$>20-30$ & 0,080 & 0,271 & 0,080 & 0,271 & 0,081 & 0,272 & 0,078 & 0,268 \\
\hline$>30-<40$ & 0,161 & 0,367 & 0,163 & 0,370 & 0,160 & 0,366 & 0,160 & 0,367 \\
\hline 40 & 0,414 & 0,493 & 0,400 & 0,490 & 0,407 & 0,491 & 0,432 & 0,495 \\
\hline$>40-45$ & 0,101 & 0,301 & 0,101 & 0,302 & 0,091 & 0,288 & 0,109 & 0,312 \\
\hline$>45$ & 0,179 & 0,383 & 0,185 & 0,388 & 0,185 & 0,388 & 0,168 & 0,374 \\
\hline
\end{tabular}


Estadísticos descriptivos. N (2008-2010) = 57.459.300 (datos elevados) (continuación)

\begin{tabular}{|c|c|c|c|c|c|c|c|c|}
\hline & \multicolumn{2}{|c|}{ 2008-2010 } & \multicolumn{2}{|c|}{2010} & \multicolumn{2}{|c|}{2009} & \multicolumn{2}{|c|}{2008} \\
\hline & Frec. & d. t. & Frec. & d. t. & Frec. & d. t. & Frec. & d. t. \\
\hline \multicolumn{9}{|l|}{ Duración de la jornada } \\
\hline Parcial & 0,141 & 0,348 & 0,140 & 0,347 & 0,152 & 0,359 & 0,132 & 0,338 \\
\hline Completa & 0,859 & 0,348 & 0,860 & 0,347 & 0,848 & 0,359 & 0,868 & 0,338 \\
\hline \multicolumn{9}{|l|}{$\begin{array}{l}\text { Convenio o estatuto específico } \\
\text { de regulación*** }\end{array}$} \\
\hline No & 0,445 & 0,497 & 0,413 & 0,492 & 0,466 & 0,499 & 0,454 & 0,498 \\
\hline Sí & 0,375 & 0,484 & 0,370 & 0,483 & 0,407 & 0,491 & 0,351 & 0,477 \\
\hline No sabe & 0,180 & 0,384 & 0,217 & 0,412 & 0,127 & 0,333 & 0,196 & 0,397 \\
\hline \multicolumn{9}{|l|}{ Puesto acorde con la formación ${ }^{\star \star \star \star}$} \\
\hline No & 0,209 & 0,407 & 0,202 & 0,402 & 0,212 & 0,409 & 0,213 & 0,409 \\
\hline Sí & 0,791 & 0,407 & 0,798 & 0,402 & 0,788 & 0,409 & 0,787 & 0,409 \\
\hline \multicolumn{9}{|l|}{ Tipo de jornada } \\
\hline Partida & 0,458 & 0,498 & 0,452 & 0,498 & 0,461 & 0,499 & 0,460 & 0,498 \\
\hline Continuada & 0,542 & 0,498 & 0,548 & 0,498 & 0,539 & 0,499 & 0,540 & 0,498 \\
\hline \multicolumn{9}{|l|}{ Tamaño del centro de trabajo } \\
\hline 1 persona & 0,109 & 0,312 & 0,107 & 0,309 & 0,111 & 0,315 & 0,110 & 0,312 \\
\hline 2-9 personas & 0,246 & 0,431 & 0,250 & 0,433 & 0,242 & 0,428 & 0,246 & 0,431 \\
\hline 10-49 personas & 0,185 & 0,389 & 0,182 & 0,386 & 0,177 & 0,382 & 0,197 & 0,398 \\
\hline 50-249 personas & 0,132 & 0,339 & 0,134 & 0,341 & 0,130 & 0,336 & 0,132 & 0,338 \\
\hline$>=250$ personas & 0,327 & 0,469 & 0,326 & 0,469 & 0,340 & 0,474 & 0,316 & 0,465 \\
\hline \multicolumn{9}{|c|}{ Actividad económica de la empresa (CNAE-09) } \\
\hline Agricultura, ganadería y pesca & 0,047 & 0,212 & 0,049 & 0,217 & 0,046 & 0,210 & 0,046 & 0,209 \\
\hline Industria & 0,152 & 0,359 & 0,145 & 0,352 & 0,148 & 0,355 & 0,163 & 0,370 \\
\hline Construcción & 0,100 & 0,300 & 0,087 & 0,282 & 0,099 & 0,298 & 0,113 & 0,317 \\
\hline Comercio & 0,143 & 0,350 & 0,145 & 0,352 & 0,143 & 0,350 & 0,143 & 0,350 \\
\hline Transporte & 0,050 & 0,217 & 0,047 & 0,211 & 0,047 & 0,211 & 0,056 & 0,229 \\
\hline Hostelería & 0,067 & 0,250 & 0,074 & 0,262 & 0,067 & 0,250 & 0,060 & 0,237 \\
\hline $\begin{array}{l}\text { Administración pública, educación } \\
\text { y sanidad }\end{array}$ & 0,226 & 0,419 & 0,226 & 0,419 & 0,236 & 0,425 & 0,217 & 0,412 \\
\hline Otros servicios & 0,214 & 0,410 & 0,227 & 0,419 & 0,215 & 0,411 & 0,202 & 0,402 \\
\hline \multicolumn{9}{|c|}{ [2008-2010] $N=39.540 .341\left(^{*}\right) N=57.335 .964\left(^{* *}\right) N=47.049 .843\left(^{(* \star}\right) N=56.172 .197\left(^{(\star \star *}\right)$} \\
\hline \multicolumn{9}{|c|}{$[2010] N=18.409 .625 N=12.912 .828\left(^{\star}\right) N=18.409 .625\left(^{(\star}\right) N=14.979 .153\left(^{(\star \star *}\right) N=17.946 .480\left(^{(\star \star \star}\right)$} \\
\hline \multicolumn{9}{|c|}{ [2009] $N=18.839 .975 N=13012794\left(^{\star}\right) N=18.717 .828\left(^{(\star *}\right) N=15.122 .239\left(^{(\star \star}\right) N=18.431 .747\left(^{(\star \star \star}\right)$} \\
\hline$[2008] N=20.209 .700 N=13.6$ & $V=20.2$ & 08.512 & **) $N=1$ & 16.948. & $51\left({ }^{* \star \star}\right) !$ & 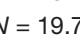 & 93.970 & $(* \star \star *)$ \\
\hline
\end{tabular}

